REVIEW ARTICLE OPEN

\title{
The yin and yang of co-inhibitory receptors: toward anti-tumor immunity without autoimmunity
}

\author{
Alexandra Schnell ${ }^{1}$, Lloyd Bod (iD), Asaf Madi ${ }^{1}$ and Vijay K. Kuchroo ${ }^{1,3}$
}

Co-inhibitory receptors are important regulators of T-cell function that define the balance between tolerance and autoimmunity. The immune regulatory function of co-inhibitory receptors, including CTLA-4, PD-1, TIM-3, TIGIT, and LAG-3, was first discovered in the setting of autoimmune disease models, in which their blockade or deficiency resulted in induction or exacerbation of the disease. Later on, co-inhibitory receptors on lymphocytes have also been found to influence outcomes in tumor and chronic viral infection settings. These receptors suppress T-cell function in the tumor microenvironment (TME), thereby making the T cells dysfunctional. Based on this observation, blockade of co-inhibitory receptors (also known as checkpoint molecules) has emerged as a successful treatment option for a number of human cancers. However, severe autoimmune-like side effects limit the use of therapeutics that block individual or combinations of co-inhibitory receptors for cancer treatment. In this review we provide an overview of the role of co-inhibitory receptors in autoimmunity and anti-tumor immunity. We then discuss current approaches and future directions to leverage our knowledge of co-inhibitory receptors to target them in tumor immunity without inducing autoimmunity.

Cell Research (2020) 30:285-299; https://doi.org/10.1038/s41422-020-0277-x

\section{INTRODUCTION}

T cells constitute a very important and potent effector compartment of the immune system. Therefore, it is critical that T-cell responses are strictly regulated to avoid inappropriate immune responses, such as autoimmune reactions. Central tolerance in the thymus acts as the first control during T-cell development to eliminate autoreactive T-cell clones. The nuclear factor AIRE expressed in medullary thymic epithelial cells facilitates ectopic expression of tissue-restricted antigens in the thymus and thereby plays an important role in the negative selection of autoreactive T cells in the thymus. ${ }^{1,2}$ The striking autoimmune phenotype in AIRE-deficient mice indicates a dominant role for central tolerance in eliminating autoreactive $\mathrm{T}$ cells and thus preventing autoimmune reactions. However, in part due to lack of self-tissue antigen expression in the thymus, altered expression of self-antigens, or low affinity expression of selfantigens, some autoreactive $T$ cells still manage to escape negative selection, leave the thymus and enter the peripheral immune repertoire. ${ }^{3}$ Hence, peripheral regulation of T-cell responses is crucial to prevent inappropriate responses to self-antigens. In the scope of this review we will focus on the role of T cell co-inhibitory molecules in the regulation of peripheral tolerance and autoimmunity, and their role in anti-tumor immunity.

\section{CO-STIMULATORY AND CO-INHIBITORY RECEPTORS}

The activation of naïve $T$ cells requires both the stimulation of the T-cell receptor (TCR) by a major histocompatibility complex (MHC)peptide complex (signal 1) and co-stimulatory signaling by costimulatory receptors (signal 2) with their corresponding ligands on antigen-presenting cells (APCs). ${ }^{4-6} \mathrm{~T}$ cell co-signaling receptors are broadly defined as cell-surface receptors that positively (co-stimulatory) or negatively (co-inhibitory) regulate TCR driven signals and therefore T-cell activation. ${ }^{6}$ As T cell co-signaling receptors have a key role in T-cell biology by directing T-cell activation, expansion and differentiation and therefore T-cell fate, the expression of these co-receptors and their ligands are strictly regulated in $\mathrm{T}$ cells and in the tissue micro-environment. An important example of a co-stimulatory pathway is the CD28:B7 axis. The co-stimulatory receptor CD28 on T cells and its ligand B71 or B7-2 on activated APCs amplify TCR signaling, leading to T-cell proliferation and IL-2 production. 6,7 To date, a number of costimulatory receptors have been identified including ICOS, CD226, OX-40, 4-1BB, and GITR. ${ }^{6}$ As $T$ cells are being activated and expanded, the expression of co-inhibitory receptors is upregulated. Multiple co-inhibitory receptors have been identified including CTLA-4, PD-1, TIM-3, TIGIT, and LAG-3. Co-inhibitory receptors play an important role in several T-cell subsets including activated T cells, regulatory $T$ cells, and exhausted $T$ cells. In activated $T$ cells, co-inhibitory receptors control and contract the expanded T-cell population. In regulatory $\mathrm{T}$ cells (Tregs), coinhibitory receptors, such as CTLA-4 and PD-1, promote the suppressive function of Tregs. ${ }^{8,9}$ In the scope of this review, we are going to focus on the role of co-inhibitory receptors on exhausted T cells. Recent work identified a critical role of T-cell exhaustion in autoimmune diseases and the targeting of co-inhibitory receptors in cancer therapy has been shown to be limited due to the development of autoimmune-like immune-related adverse events (irAEs). We are therefore interested in discussing the function of co-inhibitory receptors on exhausted $\mathrm{T}$ cells in autoimmunity versus anti-tumor immunity and leverage the recent knowledge to improve immune checkpoint blockade therapy for cancer by avoiding the induction of autoimmunity.

\footnotetext{
${ }^{1}$ Evergrande Center for Immunologic Diseases, Harvard Medical School and Brigham and Women's Hospital, Boston, MA 02115, USA; ${ }^{2}$ Department of Pathology, Sackler Faculty of Medicine, Tel-Aviv University, Tel Aviv-Yafo, Israel and ${ }^{3}$ Klarman Cell Observatory, Broad Institute of MIT and Harvard, Cambridge, MA 02142, USA

Correspondence: Vijay K. Kuchroo (vkuchroo@evergrande.hms.harvard.edu)
}

Received: 5 November 2019 Accepted: 6 January 2020

Published online: 23 January 2020 


\section{T-CELL EXHAUSTION}

T-cell exhaustion was originally discovered more than two decades ago, with the observation that virus-specific $\mathrm{CD}^{+}$ $T$ cells from mice with chronic LCMV infections lost the ability to produce effector cytokines and to mediate cytolytic effector functions. ${ }^{10}$ Loss of function during T-cell exhaustion occurs in a hierarchical manner over the course of chronic infection, with loss of both production of IL-2 and T-cell proliferation occurring early after infection. ${ }^{11,12}$ At later stages of T-cell exhaustion, virusspecific $\mathrm{CD}^{+} \mathrm{T}$ cells lose the ability to produce the cytokines IFNy and TNFa, and to degranulate. ${ }^{11,13}$ An additional key property of exhausted $\mathrm{CD}^{+} \mathrm{T}$ cells is an impaired maintenance of T-cell memory, which is controlled by the expression of Foxo $1 .{ }^{14}$ In contrast to memory $\mathrm{CD}^{+}$T cells during viral infections, exhausted $\mathrm{CD}^{+}{ }^{+} \mathrm{T}$ cells respond poorly to the cytokines IL-7 and IL-15 and are not maintained after transfer into virus-free recipient mice. ${ }^{13,15,16}$ Although best characterized in $\mathrm{CD}^{+} \mathrm{T}^{\text {cells, }} \mathrm{CD} 4^{+}$ T cells also develop T-cell exhaustion and may play a crucial part in promoting and sustaining T-cell exhaustion in $\mathrm{CD}^{+}{ }^{+}$cells. $^{17-20}$ Genomic methods including RNA-sequencing and ATACsequencing have been used to analyze the molecular pathways underlying T-cell exhaustion. ${ }^{21,22}$ These approaches allowed the identification of a molecular phenotype specific for exhausted $T$ cells, characterizing them as a distinct state of T-cell differentiation, which differs from T-cell activation or memory. The exhaustion-specific molecular changes observed in $\mathrm{CD}^{+} \mathrm{T}$ cells include altered metabolism, chemokine and chemokine receptor expression, and cytokine signaling pathways. ${ }^{21,22}$ In addition, a well-defined characteristic of exhausted $\mathrm{CD}^{+}$and $\mathrm{CD}^{+} \mathrm{T}$ cells from both human and animal models is an elevated expression of co-inhibitory receptors. ${ }^{13,22}$ Functional studies have shown an important role for inhibitory receptors in T-cell exhaustion. For example, signaling of the inhibitory receptor PD-1 following binding to its ligand PD-L1 induces T-cell exhaustion, and blocking this pathway during chronic LCMV infection restores virus-specific $\mathrm{CD}^{+}{ }^{+} \mathrm{T}$-cell responses by inducing proliferation and cytokine secretion and reduction of viral load. ${ }^{23-25}$ Furthermore, blockade of the PD-1 pathway during chronic simian immunodeficiency virus infection induces rapid expansion of virus-specific $C D 8^{+}$ $T$ cells with enhanced effector function resulting in improved survival. $^{26}$

Importantly, exhaustion has been associated with the clinical outcomes for multiple human diseases. Exhaustion correlates with persistent viraemia in a number of chronic viral infections including human immunodeficiency virus (HIV), hepatitis $C$ and $B$ virus (HCV and HBV). ${ }^{27-29}$ Interestingly, T-cell exhaustion also plays an important role in cancer and autoimmunity, albeit in opposite ways, in that T-cell exhaustion has been correlated with poor immune responses to tumors in patients and with a better prognosis in patients with autoimmune diseases ${ }^{30,31}$ (Fig. 1).

\section{T-CELL EXHAUSTION IN AUTOIMMUNITY}

$T$ cells in autoimmunity

IFNy-producing $\mathrm{CD}^{+}{ }^{+} \mathrm{T}$ cells (Th1) and IL-17-producing $\mathrm{CD}^{+}$ $T$ cells (Th17 cells) have specifically been implicated in the development of organ-specific autoimmunity. ${ }^{32}$ Originally several observations supported Th1 cells as the pathogenic subset in T cell-driven autoimmune diseases. Adoptive transfer of Th1 cells induces experimental autoimmune encephalomyelitis (EAE) with the Th1 cytokine IFNy being present in CNS lesions during EAE peak and decreased during disease remission. ${ }^{33-35}$ Mice deficient for the major Th1 transcription factors, T-bet and STAT-4, show resistance to EAE. ${ }^{36,37}$ Furthermore, administration of the Th1differentiating cytokine IL-12 aggravates collagen-induced arthritis $(\mathrm{CIA}){ }^{38}$ However, loss of the Th1-signature cytokine IFNy or STAT-1, the transcription factor that mediates IFN $y$ signaling, did not inhibit the development of autoimmunity, but paradoxically

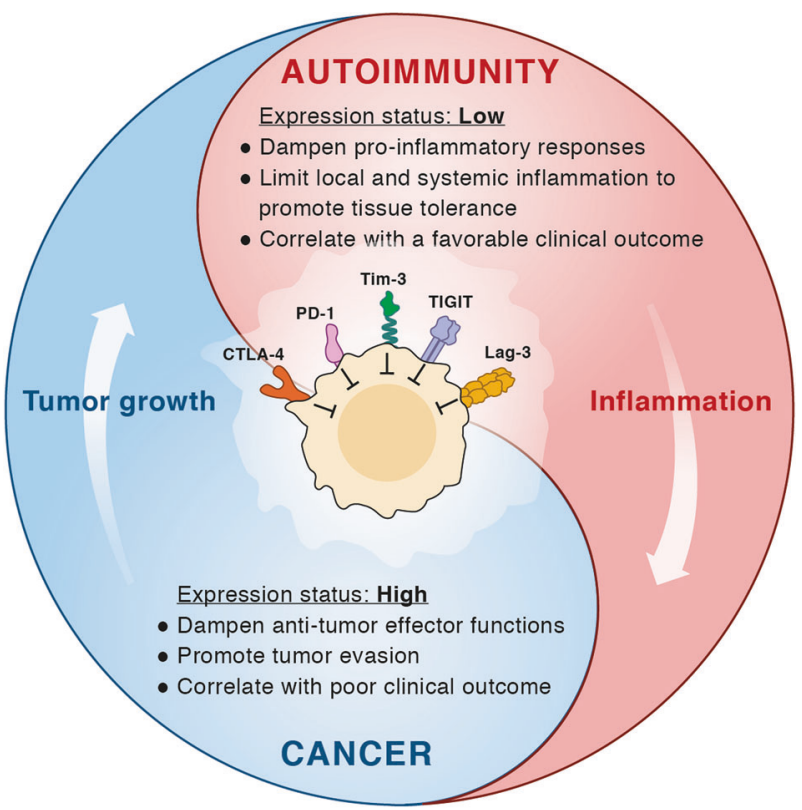

Fig. 1 The Yin and Yang of co-inhibitory receptors. Schematic representation of the co-inhibitory receptors' functional role in autoimmunity and cancer. In the tumor, co-inhibitory receptors on $\mathrm{T}$ cells dampen T-cell effector functions thereby enhancing tumor progression and correlating with worse clinical outcome. In autoimmunity, these receptors play a role in reducing local and systemic tissue inflammation, maintaining tissue tolerance, and their increased expression is associated with a good clinical outcome

enhanced autoimmune disease. ${ }^{36,39,40}$ This led to the hypothesis that there might be another subset of $T$ cells driving the development of autoimmunity and tissue inflammation. As IL-17 was found to be increased in mouse and human organ-specific autoimmune diseases, including multiple sclerosis (MS), rheumatoid arthritis, and psoriasis, research focused on the role of IL-17 producing $\mathrm{T}$ cells (named Th17 cells) as potential drivers of autoimmunity. ${ }^{41-44}$ Indeed, we and others have shown a potent role for Th17 cells in tissue inflammation and autoimmunity in multiple disease contexts. ${ }^{45}$ Th17 cells were shown to be differentiated in the presence of TGF $\beta$ and IL- 6 and further expanded or maintained by the cytokines IL- 1 and IL-23. ${ }^{46-48}$ Loss of any of the cytokines (IL-6, IL-1, IL-23) resulted in inhibition of the development of autoimmunity. ${ }^{49-51}$ Moreover, differentiation of Th17 cells in the presence of IL-23 induces more severe EAE. ${ }^{48}$ It is important to note, however, that Th1 and Th17 cells are nonmutually exclusive cell fates. IFNy and IL-17 double-positive cells have been observed in the CNS during EAE and fate mapping of IL-17 producing T cells has revealed Th17 cell plasticity to an IFN ${ }^{+}$ Th1-like phenotype. ${ }^{52-55}$ Future studies are needed to further elucidate the function of the Th17/Th1 plasticity in the pathogenesis of autoimmune diseases.

Understanding the role of T cells in autoimmunity has led to the successful development of agents for the treatment of organspecific autoimmune diseases. Monoclonal antibodies (mAbs) blocking IL-17A, secukinumab and ixekizumab, are approved for the treatment of psoriasis and secukinumab is also approved for the treatment of psoriatic arthritis and spondyloarthropathies. ${ }^{56}$ Similarly, drugs targeting the proinflammatory cytokine TNFa (infliximab, etanercept, adalimumab) have been approved for the treatment of multiple human autoimmune diseases including rheumatoid arthritis, Crohn's disease, and ankylosing spondylitis. ${ }^{57}$

However, current therapies targeting cytokines and/or cytokine signaling are not efficacious in all patients with autoimmune disease. Rather each agent must be tested in each disease, and typically not all patients diagnosed with a particular disease 
respond to treatment. Therefore, there is a need for the development of novel therapeutic approaches that will impact a common critical point that will enable efficacy in more patients and across diverse autoimmune diseases. One potential approach is to promote T-cell exhaustion by regulating expression or function of "checkpoint" molecules in autoimmune diseases.

T-cell exhaustion in autoimmunity

To identify predictive biomarkers linked to clinical outcome and novel therapeutic targets in autoimmune patients, McKinney et al. undertook an unbiased genomic analysis of $\mathrm{CD}^{+}$and $\mathrm{CD} 8^{+}$ $T$ cells from patients suffering from various autoimmune diseases. $^{31}$ The authors demonstrated that the level of T-cell exhaustion in autoimmune patients was strongly correlated with their clinical outcomes. Using independent cohorts of patients suffering from the autoimmune diseases anti-neutrophil cytoplasmic antibody-associated vasculitis (AAV), SLE, and inflammatory bowel disease (IBD), they found that for all three diseases, the T-cell exhaustion signature predicted a favorable clinical outcome. Hence, whereas T-cell exhaustion during chronic viral infections correlates with persistent viremia and worse clinical outcome, in autoimmune diseases the effect is opposite in that T-cell exhaustion seems to predict a better clinical outcome.

Additional data suggesting an important role for T-cell exhaustion in maintaining tolerance and preventing of autoimmunity has recently emerged following blockade of coinhibitory molecules to promote anti-tumor immunity. The blockade of PD-1 and CTLA-4 with mAbs in cancer patients often leads to severe irAEs in multiple tissues, including the skin, intestine, liver and lung ${ }^{58,59}$ (Fig. 2). These irAEs present pathologically like autoimmune diseases, and therefore support the concept that co-inhibitory receptors maintain peripheral tolerance, thereby preventing inappropriate autoimmune tissue inflammation.

Whereas the link between T-cell exhaustion and clinical outcomes in human autoimmunity has emerged only recently, the role of co-inhibitory molecules in autoimmune disorders has long been appreciated. In the following part of this review we will summarize the main findings highlighting the role of the coinhibitory receptors CTLA-4, PD-1, TIM-3, TIGIT, and LAG-3 in autoimmune pathology.

Co-inhibitory receptors on T cells in autoimmunity: a focus on CTLA-4, PD-1, TIM-3, TIGIT and LAG-3

The study of $T$ cell co-inhibitory receptors began with the discovery of cytotoxic T lymphocyte-associated antigen-4 (CTLA4, CD152) in 1987 as a receptor for B7 with high sequence similarity to CD28. ${ }^{60,61}$ However in contrast to CD28, CTLA-4 was found to act as a negative regulator of T-cell activation by binding to the same ligands as the co-stimulatory molecule CD28, B7-1 and B7-2, but with much higher affinity. ${ }^{62-64}$ Blockade of CTLA-4 in vitro and in vivo enhanced T-cell proliferation and effector functions. $^{65,66}$ The subsequent generation of CTLA-4-deficient mice set the stage for the discovery of the important inhibitory function of CTLA-4 in autoimmunity. Mice with germline deletion of CTLA-4 developed lethal autoimmunity characterized by lymphoblast infiltration into liver, heart, lung, and pancreas and death by 3 to 4 weeks of age. ${ }^{67,68}$ Deletion of CTLA-4 in adult mice led to spontaneous lymphoproliferation and non-lethal autoimmune disease in multiple organs. ${ }^{69}$ Moreover, in vivo blockade of CTLA-4 signaling was shown to exacerbate disease in multiple murine autoimmune models. ${ }^{70}$ For example, administration of blocking anti-CTLA-4 mAb into young diabetes-susceptible mice (BDC2.5/NOD mice) provoked rapid onset of diabetes. ${ }^{71}$ Furthermore, in relapsing-remitting $E A E$, treatment with anti-CTLA-4 mAbs enhanced disease. ${ }^{72}$ However, it is important to note that in contrast to the germline deletion, CTLA-4 deficiency in adulthood leads to the protection from EAE. ${ }^{69,73}$ These paradoxical
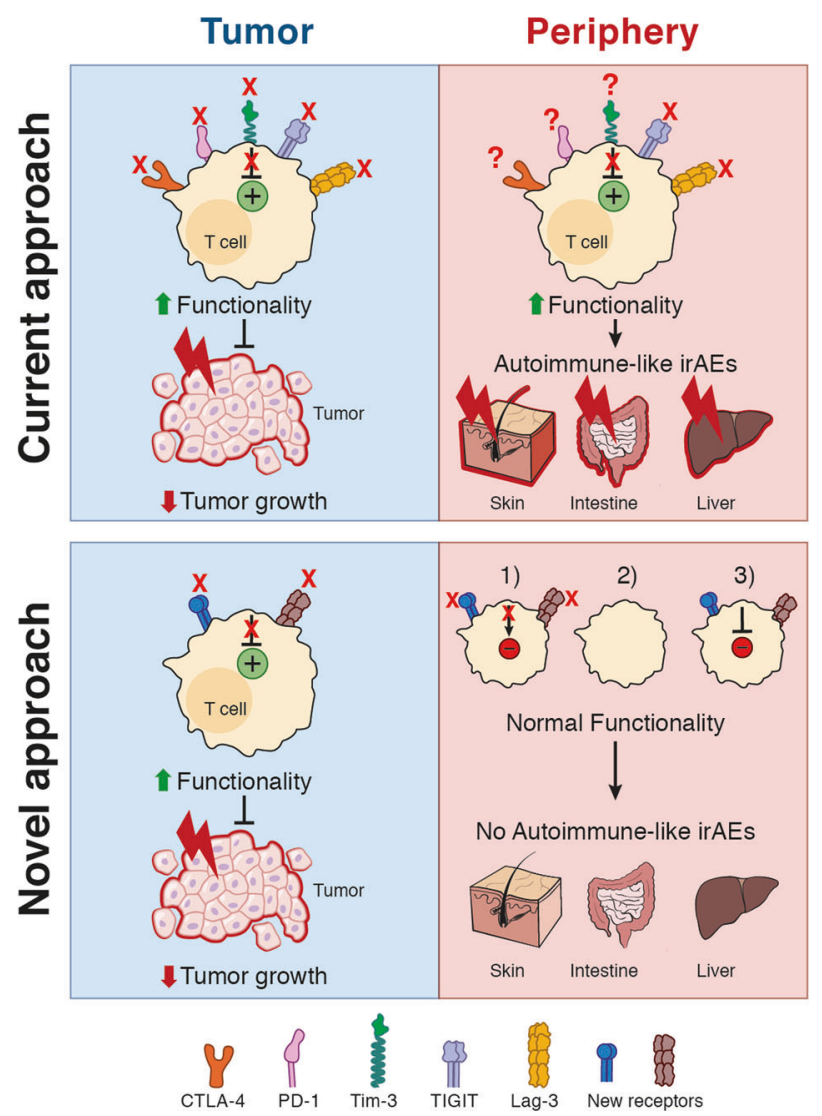

Fig. 2 Novel approaches for checkpoint blockade therapy to avoid autoimmune-like disease. Top: Current immune checkpoint blockade inhibits the signaling of co-inhibitory receptors thereby enhancing T-cell effector functions. At the tumor site, these hyperfunctional $T$ cells mediate strong anti-tumor immunity thereby reducing tumor growth (left). However, the T cells with specificity for self-antigens induce severe autoimmune-like irAEs by becoming hyper-functional and inducing tissue inflammation (right). IrAEs are mostly found in tissues with high microbial exposure, such as the skin, intestine, and liver. At homeostasis these tissues manifest a well-regulated tolerogenic environment, that is disrupted with checkpoint blockade. Bottom: Novel approaches should target new receptors mediating a potent immune response in the tumor (left) while reframing from inappropriate immune responses in the periphery against self-antigens (right). Potential mechanisms are: (1) in the periphery and in normal tissues the receptors act differentially, (2) the receptors are not expressed in the periphery, (3) the blockade only occurs in the tumor and not in the peripheral tissues

observations can be explained by compensatory immunosuppressive mechanisms following CTLA-4 deletion and an inhibitory role of CTLA-4 on Treg cells. Deletion of CTLA-4 in adult T cells led to the upregulation of inhibitory molecules including IL-10, LAG-3, and PD-1. Furthermore, the deletion of CTLA-4 specifically on Treg cells was necessary and sufficient to mediate EAE protection. ${ }^{73}$

In humans, in addition to the full-length form of CTLA-4 (fICTLA4), a soluble form of CTLA-4 exists (sCTLA-4) that lacks the transmembrane domain encoded by exon $3 .^{74,75}$ Interestingly, sCTLA-4 has been associated with type 1 diabetes (T1D). Indeed, T1D disease susceptibility mapped to an allelic variation in the $3^{\prime}$ noncoding region of CTLA-4 correlates with mRNA expression of sCTLA-4. ${ }^{75}$ However, in NOD mice, a mouse model for T1D, disease susceptibility correlates with differential expression of a different CTLA-4 splice variant, ligand-independent CTLA-4 (liCTLA-4), that lacks the B7-1/B7-2 binding domain. ${ }^{76,77}$ LiCTLA-4 was shown to be a potent inhibitor of T-cell proliferation and cytokine 
secretion. ${ }^{78}$ These different variants might occur differentially depending on the autoimmune settings, affecting the tolerance mechanisms involved.

In 1992, the second co-inhibitory receptor programmed death-1 (PD-1, CD279) was discovered and was shown to express both ITIM and ITSM signaling motifs. ${ }^{79}$ Like CTLA-4, PD1 is an inhibitory receptor on T cells that mediates its inhibitory signals via its ligands PD-L1 (B7-H1, CD274) and PD-L2 (B7-DC, CD273). PD-1 is expressed on $\mathrm{CD}^{+}$and $\mathrm{CD}^{+}{ }^{+} \mathrm{T}$ cells, $\mathrm{B}$ cells, monocytes, and subsets of dendritic cells (DCs). ${ }^{80}$ Whereas PD-L1 is broadly expressed on hematopoietic and non-hematopoietic parenchymal tissue cells, PD-L2 is only expressed on DCs and some subsets of myeloid cells. Studying PD-1 deficiency in autoimmune disease mouse models has elucidated an important role for the PD-1 pathway in autoimmune diseases. Germline deletion of $\mathrm{PD}-1$ results in development of severe autoimmune disease. Whereas BALB/C PD-1 ${ }^{-1-}$ mice develop lethal dilated cardiomyopathy, deletion of PD-1 in C57BL/6 mice results in spontaneous lupus-like autoimmune disease. ${ }^{81,82}$ T1D-prone NOD mice that are deficient for PD-1 have accelerated diabetes onset and an increased incidence of diabetes. ${ }^{83}$ Furthermore, blockade of PD-1 in EAE results in accelerated and more severe disease progression, with an increased infiltration of mononuclear cells into the CNS. ${ }^{84}$ Polymorphisms in the PD-1 locus in humans have been associated with SLE, T1D, ankylosing spondylitis, and rheumatoid arthritis. ${ }^{85-88}$

The co-inhibitory receptor $\mathrm{T}$ cell immunoglobulin and mucindomain containing protein-3 (TIM-3) was originally discovered in our laboratory as a surface protein specifically expressed on Th1 cells and IFN $\gamma$-producing $\mathrm{CD}^{+}{ }^{+}$cells. $^{89}$ As Th1 cells are established drivers of autoimmune disease, the function of TIM-3 was first studied in models of autoimmunity and it became apparent that TIM-3 mediates a potent inhibitory function. TIM-3 is expressed on $\mathrm{CD}^{+}$and $\mathrm{CD}^{+}{ }^{+} \mathrm{T}$ cells, NK cells, and myeloid cells, such as DCs and monocytes. ${ }^{90}$ The C-type lectin galectin-9 was the first ligand identified for TIM-3.91 ${ }^{91}$ Subsequently, several other ligands of TIM-3 have been identified, including phosphatidylserine and CEACAM1.92,93 Administration of anti-TIM-3 antibodies in EAE results in enhanced clinical and pathological disease scores with an increased activation phenotype in macrophages. ${ }^{89}$ Blockade of TIM-3 signaling in 2,4,6-trinitrobenzene sulfonic acid (TNBS)-induced colitis exacerbates disease, as shown by enhanced weight loss and tissue injury. ${ }^{94}$ Moreover, TIM-3 pathway blockade was observed to accelerate autoimmune diabetes. ${ }^{95}$ In addition to the results from murine autoimmune models, multiple studies also indicated an important role for TIM-3 in human autoimmune diseases. Hafler and colleagues first showed that T-cell clones generated from the cerebrospinal fluid (CSF) of MS patients produce high levels of IFNY but show decreased expression of TIM-3 compared to clones from control subjects. ${ }^{96}$ Interestingly, treatment with IFN $\beta$, an FDA-approved drug for MS, restored TIM3 expression and lessened disease activity. ${ }^{97}$ Similarly, a number of studies have shown that reductions in TIM-3 expression on $\mathrm{CD}^{+}$ and $\mathrm{CD}^{+} \mathrm{T}$ cells are inversely correlated with disease activity in patients with other autoimmune diseases, including rheumatoid arthritis, ulcerative colitis and psoriasis. ${ }^{98-100}$ In rheumatoid arthritis patients, TIM-3 expression is inversely correlated with disease activity and plasma TNFa levels. Following treatment, TIM3 expression increases and is associated with disease remission. In addition to Th1 cells, Th17 cells have been shown to express TIM3 , but at a lower level than on Th1 cells. ${ }^{101}$ In patients with psoriasis, TIM-3-negative Th1 and Th17 cells are increased in the peripheral blood, suggesting that impaired TIM-3 expression allows Th17 and Th1 cells to escape from TIM-3-mediated immunoregulation and thereby mediate disease. ${ }^{98}$ Together, these studies indicate that manipulating TIM-3 signaling in vivo may be a valuable tool in the treatment of autoimmunity. In addition to these polygenic autoimmune diseases, a recent study identified germline TIM-3 mutations in patients with subcutaneous panniculitis-like T-cell lymphoma (SPTCL). ${ }^{102}$ The mutations lead to misfolding of TIM-3 so that the protein is retained intracellularly with loss of cell surface expression. Patients harboring such loss of function mutations present with a severe autoinflammatory disease with high levels of IL-1 and TNFa in the serum and $\mathrm{CD}^{+} \mathrm{T}$ cells forming lymphomas around subcutaneous fat pads. About $30 \%$ of these patients develop autoimmune lupus-like disease characterized by elevated production of antibodies directed to double-stranded DNA. Furthermore, polymorphisms in the TIM-3 locus have been associated with multiple human autoimmune disorders. ${ }^{103}$

Another co-inhibitory molecule, T cell immunoglobulin and ITIM domain (TIGIT) was discovered as a novel member of the CD28 protein family that is specifically expressed on immune cells. TIGIT is expressed on activated T cells, a subset of Treg cells and Tfh cells, and NK cells. ${ }^{90,104-107}$ TIGIT signals via two ligands, CD155 (PVR) and CD112 (PVRL2), that are expressed on APCs, T cells, and some non-hematopoietic cells. ${ }^{90,106,107}$ Interestingly, in tumors (murine and human) both immune cells and tumor cells express high levels of TIGIT ligands. The biological function of TIGIT was initially investigated in models of autoimmunity. Mice deficient for TIGIT did not display spontaneous autoimmunity. However, the loss of TIGIT led to exacerbated autoimmune disease after immunization, or when TIGIT-deficient mice were crossed to an appropriate auto-reactive T-cell mouse line. TIGIT-deficient mice are highly susceptible to actively-induced EAE with enhanced Tcell infiltration into the CNS and increased pro-inflammatory cytokine levels. ${ }^{108}$ Furthermore, TIGIT-deficient mice crossed to the 2D2 MOG-specific TCR transgenic mice display spontaneous atypical EAE. ${ }^{108}$ Additionally, TIGIT was also found to have a protective function in $\mathrm{CIA}$. Administration of TIGIT-blocking antibodies resulted in accelerated disease onset of CIA. ${ }^{109}$ Collectively, these data suggest an inhibitory function of TIGIT on T cells in murine autoimmune models. CD226 binds to the same ligands as TIGIT, but in contrast to TIGIT, mediates a positive co-stimulatory signal, forming a network similar to the B7:CD28: CTLA4 co-stimulatory molecules. Interestingly, genome-wide association studies have linked a polymorphism in CD226 to multiple human autoimmune diseases including MS and T1D, suggesting that the TIGIT:CD226 pathway may also play a role in human autoimmunity. ${ }^{110,111}$

The co-inhibitory receptor lymphocyte activation gene-3 (LAG3) was discovered as a receptor expressed on activated T cells and a subset of NK cells. ${ }^{112}$ Interestingly, the structure of LAG-3 resembles the CD4 receptor and, in fact, LAG-3 binds to MHC class II with a higher affinity than CD4. ${ }^{113}$ In addition to MHC class II, the DC-SIGN family member LSECtin and the liver-secreted protein FGL1 have been identified as ligands of LAG-3. ${ }^{114,115}$ In contrast to mice deficient in other co-inhibitory receptors, such as CTLA- 4 and PD-1, mice deficient for LAG-3 are not susceptible to autoimmunity unless bred to a permissive genetic background. LAG-3 deficiency in B6.SJL mice results in higher susceptibility to $\mathrm{Hg}$ induced autoimmunity. ${ }^{116}$ In addition, NOD mice deficient for LAG-3 display accelerated T1D with $100 \%$ incidence. ${ }^{117}$ Interestingly, the NOD $\mathrm{LAG}^{-1-}$ mice exhibit increased T-cell numbers and enhanced proliferation of T cells in the islets, suggesting an inhibitory role for LAG-3 on T cells.

Understanding how co-inhibitory receptors are individually and collectively induced and involved in the establishment of the Tcell exhaustion state in autoimmunity would be a corner stone for their manipulation in the clinic.

Therapeutic induction of exhaustion in autoimmune diseases As T-cell exhaustion correlates with a poor outcome in cancer patients, there has been a considerable interest in targeting T-cell exhaustion to enhance immune responses to cancer cells. ${ }^{13}$ Targeting T-cell exhaustion using PD-1 blocking antibodies is a proof of concept that blockade of co-inhibitory receptors can 
restore a functional T-cell response. As mentioned earlier, in contrast to cancer, multiple lines of evidence suggest a beneficial role for T-cell exhaustion in restraining autoimmune responses, in that deficiency or blockade of inhibitory receptors results in the development of autoimmunity in mice, and induction of autoimmunity is observed in humans during immune checkpoint blockade therapy for cancer. Additionally, a transcriptional signature of T-cell exhaustion is associated with a favorable clinical outcome in multiple human autoimmune diseases. ${ }^{31}$ Based on these data, promoting T-cell exhaustion could be beneficial in autoimmunity, and provides a novel therapeutic approach for inhibiting autoimmunity ${ }^{31,118}$ (Fig. 3 and Table 1). Agonist antibodies and Fc-fusion proteins that engage co-inhibitory receptors have been successful in treating murine models of autoimmunity. ${ }^{119}$ For example, cells from the draining lymph nodes of mice treated with agonistic anti-TIGIT antibodies showed reduced cell proliferation and proinflammatory cytokine production after restimulation with MOG-peptide. Moreover, the severity of EAE was reduced in treated mice accompanied by decreased frequencies of IL-17-producing lymphocytes in the CNS. ${ }^{120}$ The administration of PDL-1.Fc to crosslink PD-1 ameliorated the severity of $\mathrm{CIA}$, which was accompanied by decreased proliferation of T cells. ${ }^{121}$ Furthermore, administration of the TIM-3 ligand Galectin-9 decreased CIA severity. ${ }^{122}$ However, these studies did not prove that the agonistic agents directly induce signaling through the co-inhibitory receptors. Hence, future mechanistic studies will be required for translating the use of compounds triggering co-inhibitory receptors for the treatment of autoimmune diseases to the human setting.

Moreover, in order to facilitate the induction of T-cell exhaustion as therapy for human autoimmune diseases, a better understanding of the underlying biology of T-cell exhaustion is required. In a recent study, our laboratory used unbiased RNA and protein expression profiling to identify a module of co-inhibitory receptors in $\mathrm{CD}^{+}$and $\mathrm{CD}^{+}{ }^{+}$cells. ${ }^{123}$ Interestingly, our studies identified the immunoregulatory cytokine IL-27 as a key inducer of the co-inhibitory gene module. In addition, the transcription factors PRDM1 and C-MAF, induced by IL-27, were identified as cooperative regulators of the co-inhibitory gene module. Loss of both PRDM1 and c-MAF resulted in an about 2-fold decrease in expression of the co-inhibitory genes accompanied by increased anti-tumor immunity. Additional studies using novel unbiased computational methods are going to be required in the future to identify and test mediators of T-cell exhaustion in the treatment of autoimmune diseases. ${ }^{124}$ Furthermore, a better analysis of the tissue-specific molecular and cellular pathways inducing T-cell exhaustion remains mandatory in order to improve its targeting.

\section{T-CELL EXHAUSTION IN CANCER}

$T$ cells in tumor immunity

Historically, cancer research has been dominated by a tumorcentric view, deciphering intrinsic features of tumor cells. However, Ehrlich's theories, Coley's clinical observations and experimental studies from Macfarlane Burnet and Lewis Thomas provided a strong rationale for cancer immunosurveillance. ${ }^{125-129}$ In recent years, the concept of tumor cells interacting with the immune system and other cell types has become the focus of a number of studies. Among the cell types composing the TME, $T$ cells have gained the most attention in cancer research. Tumor cells harboring genetic alterations differ from normal cells and are able to induce tumor-reactive T-cell responses. ${ }^{130-134}$ During the T-cell response to cancer, tumor antigen-experienced lymphocytes undergo activation and differentiation into effector and memory fates. ${ }^{135-137}$ Heterogeneity among these two cell fates has been demonstrated, with the description of multiple effector and memory subsets. ${ }^{137-139}$ T cells are present in the TME of most solid tumors and it is well established that infiltration of T cells, particularly $\mathrm{CD}^{+} \mathrm{T}$ cells, correlates with a positive clinical outcome in several cancer types. ${ }^{140,141} \mathrm{CD}^{+} \mathrm{T}$ cells have the ability to engage various differentiation pathways including the Th1-type pathway that may have a direct anti-tumor role via the secretion of IFNy or TNFa. However, the most notable effect of $\mathrm{CD}^{+} \mathrm{T}$ cells is to provide help to expand and differentiate CD8 ${ }^{+}$

\section{A U TOIM M U N ITY}

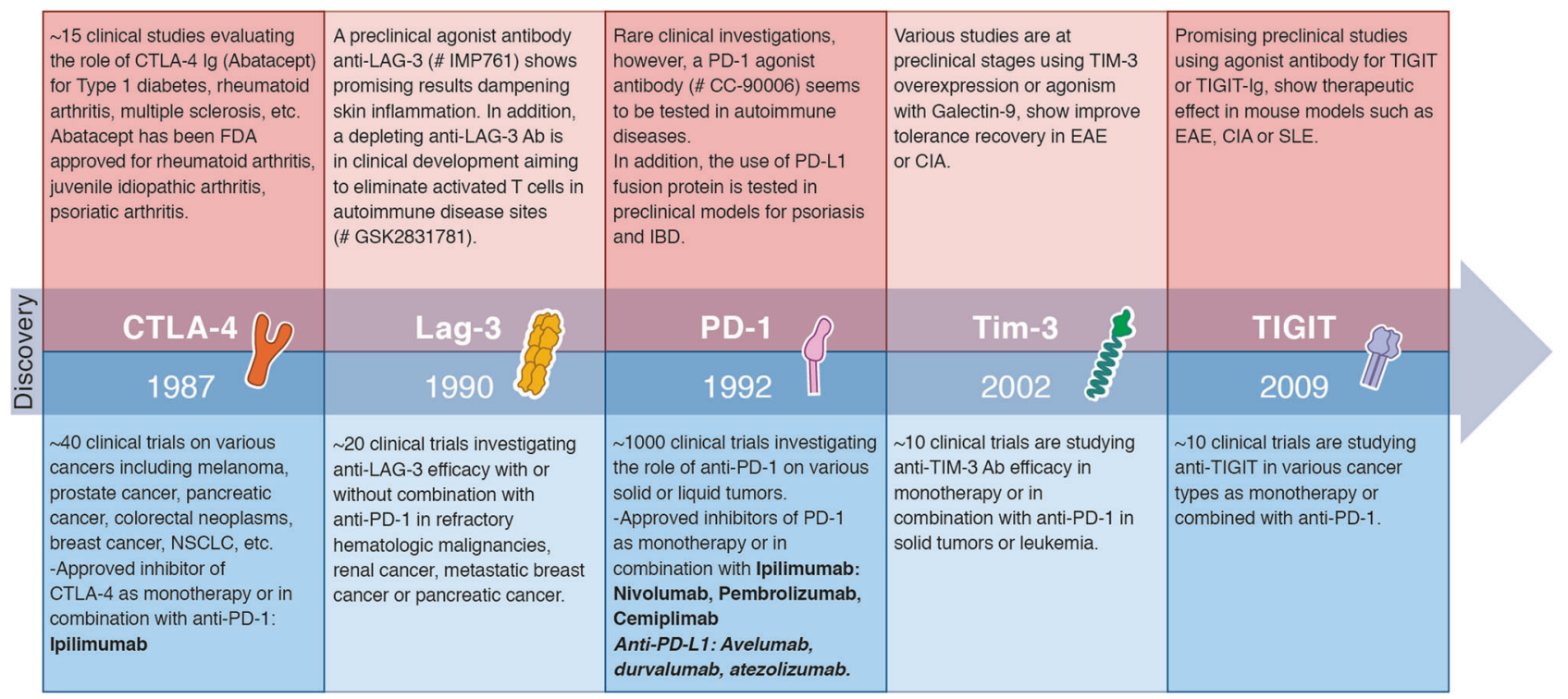

\section{A N C E R}

Fig. 3 Therapeutic targeting of co-inhibitory receptors in autoimmunity and cancer. Timeline of discovery and therapeutic targeting of the five co-inhibitory receptors discussed in this review: CTLA-4, ${ }^{60}$ LAG-3, ${ }^{12}$ PD- $1,{ }^{79} \mathrm{TIM}-3,{ }^{89}$ TIGIT. ${ }^{106}$ The clinical or preclinical investigations and FDA-approved drugs are listed for both autoimmunity (red) and cancer (blue) according to https://clinicaltrials.gov and https://www.fda.gov 
Table 1. Co-inhibitory receptors in autoimmunity and cancer

\begin{tabular}{|c|c|c|}
\hline Inhibitory receptor & Autoimmunity & Cancer \\
\hline $\begin{array}{l}\text { TIGIT (T cell immunoglobulin and } \\
\text { ITIM domain) }\end{array}$ & $\begin{array}{l}\text { - Global knockout: Highly susceptible to } \mathrm{EAE}^{108} \\
\text { - In vivo blockade: Accelerated disease onset of } \mathrm{CIA}^{109} \\
\text { - Human disease association: Polymorphism in } \mathrm{CD}^{226} \\
\text { linked to multiple human autoimmune diseases }\end{array}$ & $\begin{array}{l}\text { - Global knockout and conditional knockout in Tregs } \\
\text { but not CD8 } 8^{+} \text {cells reduce tumor growth in vivo } \\
\text { - In vivo blockade: Synergized with anti-PD-1 treatment } \\
\text { leading to tumor regression }{ }^{120,196} \\
\text { - Clinical studies showing beneficial therapeutic impact } \\
\text { of TIGIT blockade or co-blockade with PD-1 in } \\
\text { multiple cancer types }{ }^{196,201,202}\end{array}$ \\
\hline $\begin{array}{l}\text { LAG-3 (Lymphocyte activation } \\
\text { gene-3) }\end{array}$ & $\begin{array}{l}\text { - Global knockout: No increased susceptibility to } \\
\text { autoimmune disease unless crossed to permissive } \\
\text { genetic background. LAG3-deficient NOD mice } \\
\text { accelerated T1D, }{ }^{117} \text { LAG3-deficient B6.SJL mice } \\
\text { higher susceptibility to Hg-induced autoimmunity }{ }^{116}\end{array}$ & $\begin{array}{l}\text { - Global knockout or in vivo blockade reduces the } \\
\text { growth of transplantable tumors }{ }^{203} \\
\text { - Strong synergistic effect with PD-1 to promote tumor } \\
\text { progression }{ }^{209-212} \\
\text { - Encouraging clinical trials evaluating LAG-3-targeted } \\
\text { therapies in cancer patients }{ }^{272}\end{array}$ \\
\hline
\end{tabular}

T cells into cytotoxic T lymphocytes (CTLs). With the help of DCs, $\mathrm{CD}^{+} \mathrm{T}$ cells are able to recognize and lyse tumor cells via the duo granzyme B/perforin, FasL and TRAIL molecules. However, as described above in chronic infections and cancer, a significant fraction of antigen-specific $\mathrm{CD}^{+}{ }^{+} \mathrm{T}$ cells attain a hypo-responsive state called T-cell "exhaustion".

T-cell exhaustion in tumor immunity

T-cell exhaustion was a term first coined in the context of chronic viral infection, as a state of dysfunctional phenotype where T cells progressively lose their effector function due to chronic antigen exposure, further promoted by a lack of $\mathrm{CD}^{+} \mathrm{T}$-cell help and exposure to immunosuppressive cytokines. ${ }^{22,142}$ This concept expanded to the field of cancer research due to high tumorantigen loads and the tolerogenic TME. T cells isolated from human tumors or murine models were initially described as phenotypically and functionally similar to exhausted $T$ cells described in chronic infections, however there might be nuanced differences between those exhausted T cells found in chronic viral infections and the ones found in the TME. ${ }^{143,144}$ However, for the purpose of this review the dysfunctional T cells found in TME will be referred to as exhausted $T$ cells. Exhausted $T$ cells are characterized as a fraction of tumor-reactive $\mathrm{CD}^{+} \mathrm{T}$ cells that are unable to lyse tumor cells, and have impaired effector functions including production of potent effector cytokines (e.g., TNFa, IFN $\gamma$, IL-2) together with high expression of co-inhibitory receptors including CTLA-4, PD-1, TIM-3, TIGIT or LAG-3. ${ }^{13,21,22,145}$ More recently, there has been an increasing focus on the transcriptional regulators that initiate, amplify or maintain the exhaustion state. Singer et al. have identified different gene programs regulating $\mathrm{CD}^{+}$tumor-infiltrating lymphocytes (TILs) dysfunction. The zinc-finger transcription factor GATA-3 was highlighted to be one of the drivers of the dysfunctional state in those cells. ${ }^{123,145}$ A recent publication by Vodnala et al. showed that the TME nutrients and metabolites milieu, especially potassium concentrations, impact the T-cell genome and epigenome, triggering T-cell stemness. ${ }^{146}$ Moreover, the exhaustion-fate commitment of $\mathrm{CD}^{+} \mathrm{T}$ cells has been recently attributed to the expression of the HMG-box transcription factor TOX, which appears to be critical for initiating the exhaustion epigenetic program in both chronic viral infections and cancer. ${ }^{147-149}$ Dissecting the exhaustion state and transcriptional program remains essential in order to improve the identification and targeting of exhausted T cells in cancer. Expression of inhibitory receptors present on the surface of exhausted T cells form a functional module which is tightly controlled within the TME. ${ }^{123,145}$ In line with the previous section regarding the role of checkpoint receptors in autoimmunity, we will explore several inhibitory receptors and will highlight rationale, mechanisms and recent advances from both experimental models and clinical data emphasizing the value of those receptors as potential targets to manipulate for cancer therapy. 
Co-inhibitory receptors on $T$ cells in anti-tumor immunity: a focus on CTLA-4, PD-1, TIM-3, TIGIT and LAG-3

Promptly after their discovery, investigations aiming to target coinhibitory receptors arose and established a new paradigm in therapies for cancer. As previously described, CTLA-4 acts as a brake on T-cell responses. In cancer, CTLA-4 is expressed on activated $\mathrm{CD}^{+}$and $\mathrm{CD}^{+}{ }^{+} \mathrm{T}$ cells and on Treg cells. ${ }^{9,150,151}$ CTLA-4 can also be expressed by tumor cells themselves. ${ }^{152}$ Unfortunately, the rapid and fatal autoimmunity in CTLA-4-deficient mice limits the analysis of experimental tumor models. ${ }^{67,68}$ Interestingly, the inducible deletion of CTLA-4 expression in adult mice has no effect on the growth of MC38 colon adenocarcinoma cells in vivo, raising questions regarding the mechanisms involved in CTLA-4 targeting therapies. ${ }^{73}$ The first evidence that fostered enthusiasm in the field was the in vivo blockade of CTLA-4, published in 1996, leading to a remarkable control of tumor growth in mice. ${ }^{66}$ Notably, anti-CTLA-4 treatment restored exhausted T-cell effector functions, but the therapeutic effect was mainly due to the Fc-dependent depletion of Tregs occurring after anti-CTLA-4 administration. ${ }^{153-156}$ Moreover, conditional deletion of CTLA-4 specifically in Tregs revealed important insights into their ability to promote tumor growth. ${ }^{9}$ In human cancer, several studies have described a negative correlation between the levels of CTLA-4 mRNA or protein and the clinical outcome in both leukemic and solid tumors. ${ }^{157-159}$ Interestingly, in non-small cell lung cancer (NSCLC), high CTLA-4 expression in primary tumors predicts an improved patient survival, whereas its expression in the sentinel lymph node correlates with a poor clinical outcome. ${ }^{160}$ Multiple studies identified SNPs within the CTLA-4 locus associated with various effects on cancer outcome. ${ }^{161}$ In particular, some SNPs in the promoter region of the CTLA-4 locus that regulate its expression, have been associated with higher susceptibility to pancreatic cancer or breast cancer. ${ }^{162,163}$ These data pave the way for improved personalized medicine with a deeper screening of these variants allowing the adjustment of targeted therapies.

PD-1 was initially identified as a receptor on T cells associated with programmed cell death. ${ }^{79}$ Further studies led to evidence for its role in anti-tumor immune responses. Its ligands, PD-L1 and PDL2 expressed on various tumors and myeloid cells, dampen antitumor immune responses. ${ }^{164-166}$ Interestingly, tumor-derived PD$\mathrm{L} 1$ is sufficient to directly inhibit $\mathrm{CD}^{+} \mathrm{T}$ cells, thereby promoting tumor escape. ${ }^{167}$ Moreover, blockade, germline knockout or specific deletion of PD-1 in T cells leads to accelerated tumor clearance in multiple mouse models. ${ }^{168-171}$ In several types of cancer, the expression of PD-1 and/or its ligands is elevated and has been associated with poor prognosis and is predictive of response to antibodies targeting the PD-1 pathway. ${ }^{172,173}$ Several studies have also identified PD-1 polymorphisms and their association with susceptibility to various cancer types. ${ }^{174}$ These studies have led to the successful therapeutic targeting of CTLA-4 and PD-1 for various malignancies.

Beyond these two receptors, preclinical data support a role for three other co-inhibitory receptors, TIM-3, TIGIT, and LAG-3, in cancer. Expression and function of TIM-3 was rapidly deciphered in cancer biology, due to its role in the regulation of type 1 immune responses. ${ }^{89,91,175-177}$ TIM-3 was found to be highly expressed by $\mathrm{CD}^{+} \mathrm{PD}-1^{+} \mathrm{T}$ cells which include the most dysfunctional subset of tumor-infiltrating lymphocytes in tumors. $^{178,179}$ TIM-3 overexpression on $\mathrm{T}$ cells leads to the expansion of myeloid-derived suppressor cells and promotes tumor growth. ${ }^{175}$ In contrast, the blockade of TIM-3 reduces tumor growth and ameliorates tumor-specific $\mathrm{CD}^{+}$T-cell responses. ${ }^{179,180}$ High TIM-3 expression was shown to be associated with a poor prognosis in multiple human cancers such as colon, gastric, non-small cell lung, and clear cell renal carcinoma. $^{181}$ In human colorectal cancer patients, high TIM-3 concentration correlates with disease progression. ${ }^{182}$ Several TIM3 polymorphisms have been reported to be associated with a higher risk of digestive cancer, renal cell carcinoma, pancreatic cancer and non-small cell lung cancer. ${ }^{183-186}$ At least 9 different clinical trials investigating TIM-3 inhibition in solid tumors show promising results, especially in combination with PD-1 blockade $^{187}$ (Clintrials.gov).

TIGIT is highly expressed on NK cells, CD8 ${ }^{+} \mathrm{T}$ cells and Treg cells within the TME. ${ }^{188-191}$ Its ligands CD155 and CD112 are widely expressed on tumor cells. ${ }^{192-194}$ Multiple studies showed that TIGIT signaling is involved in dampening both T-cell activation and differentiation into anti-tumor effector cells. ${ }^{107}$ Interestingly, the majority of tumor-infiltrating Tregs expresses TIGIT, and TIGITdeficient Tregs, but not $\mathrm{CD}^{+} \mathrm{T}$ cells, are able to reduce tumor growth in vivo. ${ }^{195}$ More recently, blocking anti-TIGIT antibodies synergized with anti-PD-1 treatment leading to tumor regression in vivo. ${ }^{120,196}$ In cancer patients, TIGIT expression has been associated with a poor clinical outcome in liquid or solid tumors. ${ }^{197-200}$ Currently, a number of clinical studies are investigating the therapeutic impact of TIGIT blockade either alone or in combination with PD-1 in multiple cancer types. ${ }^{196,201,202}$

The checkpoint receptor LAG-3 holds great potential in cancer immunotherapy. LAG-3 is expressed widely in tumor-infiltrating lymphocytes in various tumors. ${ }^{203-205}$ Initially described to modulate anti-tumor cell cytotoxicity in vitro, ${ }^{205-208}$ the blockade or germline deletion of LAG-3 reduces the growth of transplantable tumors in vivo. ${ }^{203}$ Remarkably, LAG-3 synergizes strongly with PD-1 to promote tumor progression. ${ }^{209-212}$ Ongoing investigations are highlighting new ligands for LAG-3 (e.g., galectin-3 or Fibrinogen-like protein 1) that are present in the TME together with novel immunomodulatory functions that will consolidate the basis for future therapeutic development of LAG-3 targeting reagents. Particularly, the high affinity interaction of Fibrinogen-like protein 1 (FGL1) and LAG-3 has revealed a major immune-evasion mechanism suppressing $T$ cell anti-tumor responses and may constitute an important targetable pathway in cancer immunotherapy. ${ }^{115} \mathrm{~A}$ comprehensive appreciation of the receptor-ligand interactions and their biology remains essential for an optimal therapeutic targeting in clinic.

\section{Manipulating T-cell exhaustion in cancer}

Checkpoint blockade-based immunotherapy. Based on initial studies on mouse tumors, anti-CTLA- 4 antibodies were tested in clinical trials for human cancers. In 2010, targeting CTLA-4 pioneered checkpoint blockade-based immunotherapy by improving the survival of patients with metastatic melanoma. ${ }^{213}$ A year after the publication of the clinical trial, anti-CTLA-4 immunotherapy was approved by the FDA for advanced melanoma, paving the way for targeting of other checkpoint receptors such as PD-1 in 2014. However, the response rate of patients to these monotherapies remained modest. For example, in patients with metastatic melanoma, the objective response rate (ORR) for ipilimumab (anti-CTLA-4) was about 10\%-16\% and for nivolumab (anti-PD-1) was $30 \%-40 \%$. Combinations of agents targeting CTLA-4 and PD-1 were thus investigated to increase the ORR and survival rates of patients. Indeed, in several studies, combination of ipilimumab and nivolumab increased the ORR to about $60 \%$ in various cancers, ${ }^{214-218}$ reaching $90 \%$ ORR in melanoma. ${ }^{214,219}$ However, some tumors were refractory or acquired tumor-resistance to these therapies preventing clinical success. $^{220}$ Furthermore, a significant fraction of patients developed severe irAEs, especially with combined CTLA-4 and PD-1 blockade, increasing the clinical need to optimize these therapies. $^{221}$ Multiple investigations of optimized therapies (combination with other therapies, treatment kinetics, specificity, various lgisotypes or Fc-engineered antibodies) aim to improve the efficacy while mitigating the toxicity of anti-PD1 and anti-CTLA-4 antibodies. ${ }^{222-224}$ Interestingly, a recent study showed that in combination with vaccines against tumor-associated antigens, the timing of anti-PD-1 blockade influences therapeutic outcome. Particularly, PD-1 blockade under suboptimal priming conditions 
leads to the emergence of a subset of dysfunctional $\mathrm{CD}^{+} \mathrm{T}$ cells that prevent antitumor immunity. ${ }^{225}$ However, these observations have catalyzed exploration into novel co-inhibitory molecules expressed on exhausted $T$ cells with the goal of identifying receptors with more tumor-restricted expression to achieve increased efficacy while limiting autoimmune-like toxicity. Multiple clinical trials are currently investigating the use of TIM-3, TIGIT and LAG-3 as targets for monotherapy or in combination with anti-PD-1 and have shown promise for cancer therapy $90,217,218,226$ (Fig. 3 and Table 1).

Checkpoint-inhibitor induced immune-related adverse events (irAEs). As mentioned above, perturbing immune exhaustion or tolerance can provoke inappropriate autoimmune reactions (Fig. 2). Following checkpoint blockade, these overexuberant responses lead to irAEs, which could derive from: 1. an aggravation of a silent preexisting autoimmune condition 227,$228 ; 2$. a neo-autoimmune or inflammatory disorder because of breaking self-tolerance; 3 . a disruption of immune homeostasis in tissues; 4 . a bystander selftissue damage (on target/off tumor responses); or 5. undesired reactions to the checkpoint blockade (e.g., expression of coinhibitory molecules on non-T cells). The most common irAEs after checkpoint blockade are dermatologic (47\%-65\%), colitis $(30 \%-48 \%)$, hepatitis $(5 \%-30 \%)$ and/or endocrine $(5 \%-10 \%)$ with different grades of severity. ${ }^{229,230}$ Interestingly, irAEs are more frequent in cancer patients upon anti-CTLA-4 $(60 \%-85 \%$, mostly grades 1 and 2$)$ than anti-PD-1 (16\%-37\%, with a minority of patients displaying high-grade toxicity) blockades. 229,231-233 Environmental factors have been shown to influence the occurrence of irAEs. For example, the microbiome has been shown to affect efficacy and immunotoxicity of checkpoint blockade. Dysbiosis induced by antibiotics was revealed to negatively impact on the clinical outcome of cancer patients who were treated with checkpoint blockade therapies. ${ }^{234-236}$ Additional factors found to influence susceptibility to irAEs include the patients' genetics/ epigenetics ${ }^{237}$ and autoimmunity related to variants in the major histocompatibility complex (MHC) locus. ${ }^{238} \mathrm{~A}$ careful establishment of a risk score specific to each immunotherapy may provide important insights that can be used by clinicians to improve immunotherapeutic strategies while limiting irAEs.

\section{TOWARD TUMOR IMMUNITY WITHOUT AUTOIMMUNITY}

Despite the impressive efficacy of immune checkpoint blockade in the treatment of some cancers, the manifestation of autoimmune disease-like irAEs has become a critical limitation for the applicability of these drugs in clinic. Additionally, many patients fail to respond to current checkpoint blockade therapies. Current and future efforts in the field are directed toward promoting tumor-specific immunity with greater efficacy in more clinical settings with more tolerable side effects (Fig. 2).

Shared features of exhausted tumor-infiltrating $T$ cells and proinflammatory $\mathrm{CD}^{+}{ }^{+} \mathrm{T}$ helper cells

As discussed in an earlier section, Th1 and Th17 cells have been implicated in the pathogenesis of multiple autoimmune diseases. Our laboratory and others have shown that Th17 cells come in multiple phenotypes. ${ }^{239-241}$ Th17 cells differentiated by TGF- $\beta 1$ and IL- 6 produce IL-17 and IL-10 and do not mediate tissue inflammation, thus are referred to as "non-pathogenic" Th17 cells. ${ }^{241}$ In contrast, supplementation of IL-23 to the differentiation medium leads to the generation of "pathogenic" Th17 cells. ${ }^{240}$ These "pathogenic" Th17 cells drive inflammation in multiple autoimmune disease mouse models. ${ }^{239,242}$ Using microarray analysis and single-cell RNA-seq of Th17 cells, our laboratory identified a unique transcriptional signature of pathogenic Th17 cells. $^{53,239}$ Interestingly, when comparing the transcriptional signature of exhausted T cells to Th17 cells, it is evident that a noteworthy signature overlap exists ${ }^{53,123,239}$ (Fig. 4). Genes that are upregulated in exhausted T cells in cancer and in Th17 cells in autoimmunity might play an important functional role in both settings. Over the last few years, our laboratory successfully validated the functional role of some of these molecules in cancer as well as in autoimmunity. For example, the transmembrane protein podoplanin (PDPN) is highly expressed on Th17 cells and acts as a negative regulator of Th17 pathogenicity. ${ }^{243}$ Mice with a selective depletion of PDPN in T cells show enhanced EAE severity with increased T-cell infiltration into the CNS. ${ }^{244}$ Interestingly, PDPN was also identified as an important regulator in cancer. PDPN is part of the co-inhibitory gene module upregulated in cancer, and PDPN deficiency in T cells results in retardation of tumor growth. ${ }^{123}$ The protein $C$ receptor (PROCR) is another molecule highly expressed in Th17 cells and exhausted T cells. ${ }^{123,245}$ In autoimmunity, PROCR was found to act as a negative regulator of Th17 pathogenicity. ${ }^{245}$ PROCR regulated the pathogenic gene module of Th17 cells by modulating expression of IL-1R, a major driver of pathogenic Th17 cells, and T cell-specific deficiency of PROCR resulted in exacerbated EAE. In the tumor setting, PROCR deficiency inhibited tumor growth with decreased frequencies in exhausted (TIM-3 $3^{\text {High }}$ and PD- ${ }^{\text {High }}$ ) $\mathrm{CD}^{+} \mathrm{T}$ cells. ${ }^{123}$ Another example is the glycosphingolipid receptor GPR65 that was identified in vivo to co-vary with pro-inflammatory genes in single-cell RNA-sequencing of Th17 cells during EAE. ${ }^{53}$ Interestingly in contrast to PDPN and PROCR, GPR65 was validated as pathogenic driver of Th17 cells in EAE. Mice deficient in GPR65 were found to be protected from EAE and showed decreased IL17A- and IFNy-positive cells in the spleen and lymph nodes when compared to wildtype mice. Interestingly, GPR65 was additionally identified to be part of the co-inhibitory receptor module driven by IL-27. ${ }^{123}$ In future studies, additional genes that are shared between proinflammatory Th1 and Th17 signatures and the exhausted T-cell signature may provide a novel set of targets that might differentially impact generation of pathogenic Th17 cells and thereby impact autoimmunity but at the same time may affect development of T-cell exhaustion and promote anti-tumor immunity (Fig. 4). Targeting these molecules has the potential to simultaneously yield benefits for tumor immunity without triggering autoimmune side-effects.

Tumor-specific T-cell signatures

In order to target $\mathrm{T}$ cells specifically in the tumor, it will be important to precisely determine the differences between $T$ cells in tumors and those at other tissue sites. Using next generation technologies (e.g., RNA-sequencing, ATAC-sequencing), it is possible to unbiasedly define tumor-specific signatures both in effector cells (CD4 ${ }^{+}$and $\mathrm{CD}^{+} \mathrm{T}$ cells), regulatory $\mathrm{T}$ cells, and myeloid cells. In a recent multi-pronged study carried out by Magnuson et al., the authors discovered a specific transcriptional profile of tumorinfiltrating Tregs across species and tumor types. ${ }^{246}$ This study identified multiple candidate genes encoding possible new targets for cancer immunotherapy. Using a CRISPR-based approach, the authors validated some of those targets as potential modulators of Treg infiltration and function in the tumor. The loss-of-function mutations of TNFRSF8, CXCR3, and SAMSN1 resulted in reduced frequencies of tumor-infiltrating Tregs. Similar studies combining computational, molecular, and functional systems aimed to precisely define a tumor specific $\mathrm{CD}^{+}{ }^{+}$-cell exhaustion gene signature in order to discover potential new targets for immunotherapies. ${ }^{145,247}$ A recent study identified a subpopulation of "progenitor exhausted" CD8 ${ }^{+}$T cells, carrying a unique genetic and epigenetic signature and being able to respond better to anti-PD-1 therapy. In melanoma patients, the frequency of these progenitor exhausted cells positively correlates with the duration of response to checkpoint-blockade therapy. ${ }^{248}$ These progenitors are characterized by the expression of the transcription factor TCF-1, which sustains stemness and controls effector differentiation. Interestingly, 


\section{Cancer exhaustion signature}

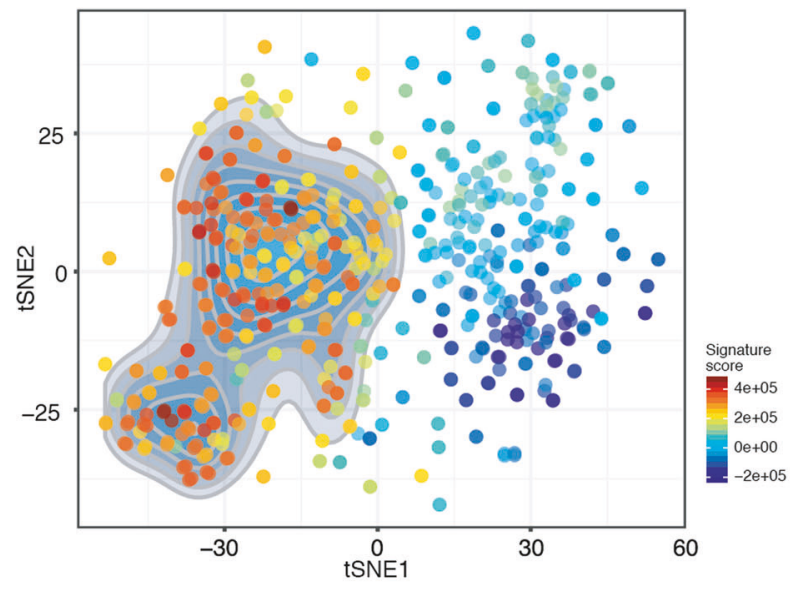

\section{Th17 pathogenic signature}

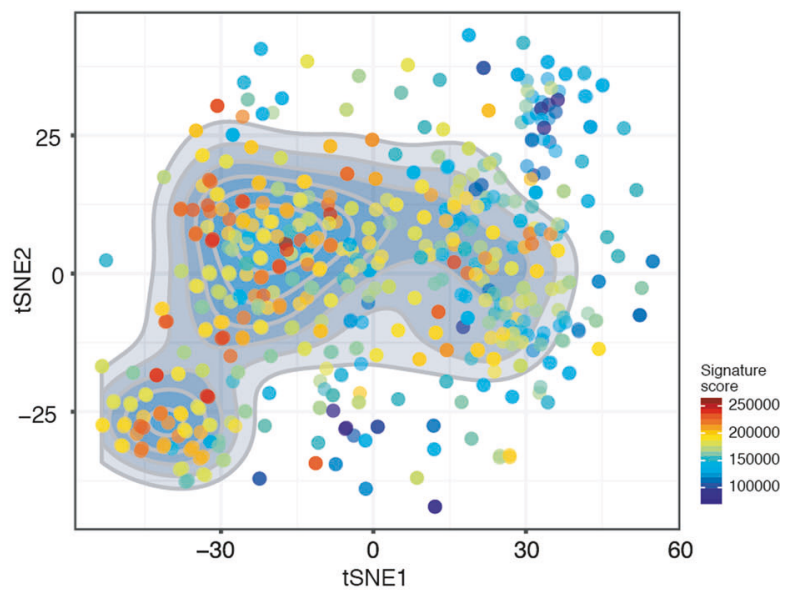

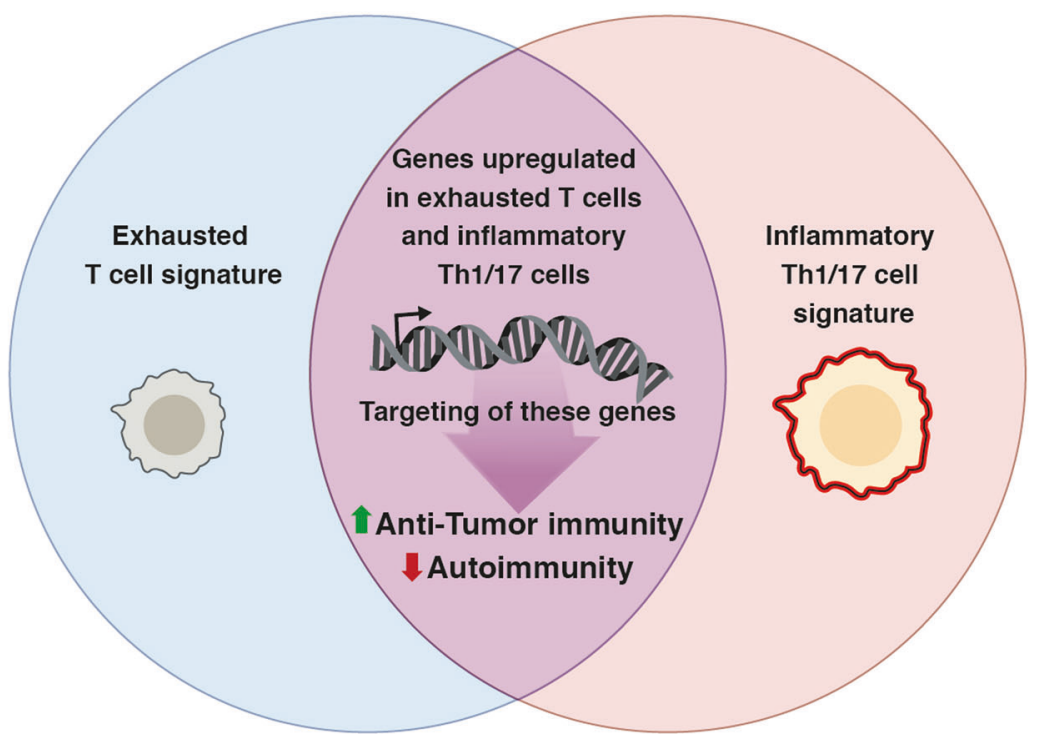

Fig. 4 Shared transcriptional signature of inflammatory Th1 and Th17 cells and exhausted T cells. Pathogenic Th17 cells are established drivers of multiple autoimmune diseases. Exhausted T cells are hypo-functional T cells preventing active tumor immunity. Both T-cell states share part of their transcriptional signature. Interestingly, when projecting the cancer exhaustion signature ${ }^{249}$ and the pathogenic Th17 signature ${ }^{239}$ on the tSNE plot of single-cell CD8 ${ }^{+}$TILs (top), multiple single cells show enrichment for both signatures, suggesting that shared modules or transcriptional programs are activated in those cells. Members of this shared signature could play a key role in T-cell activation and later exhaustion. Targeting of these genes could potentially yield in an enhanced anti-tumor immunity without increased autoimmunity

a strict antagonism between TCF-1 and TIM-3 expression exists, with TCF-1 marking stem-like progenitor cells and TIM-3 marking terminally exhausted $T$ cells that fail to be reinvigorated by immune checkpoint blockade. ${ }^{249-254}$ A better understanding of this potential inverse relationship between TCF-1 and TIM-3 would be critical to manipulate T-cell state and strengthen antitumor responses upon immunotherapies. Having a clearer definition of tumor-specific T-cell phenotypes will allow the design of more tumor-restricted immunotherapies, decreasing uncontrolled responses and limiting autoimmune-like toxicity.

Tumor-specific checkpoint blockade

An additional idea to improve checkpoint blockade is to leverage the efficacy of the current immune checkpoint blockade drugs while limiting the manifestation of irAEs at non-tumor sites. In a recent study by Perez-Ruiz et al., the authors show that prophylactic blockade of TNF ameliorates colitis but enhances tumor immunity in mice. ${ }^{255}$ TNF blockade is a well-established treatment option for multiple autoimmune diseases, including rheumatoid arthritis, psoriasis and $\mathrm{IBD}^{256}$ Furthermore, TNF blockade is used in patients developing irAEs following immune checkpoint blockade that are refractory to steroids. ${ }^{257}$ In this study the authors show that the dual treatment with anti-CTLA-4 and anti-PD-1 mAbs worsens DSS-induced colitis. Interestingly, prophylactic blockade of TNF strikingly ameliorates the treatmentinduced worsening of the colitis. Moreover, prophylactic TNF blockade does not hinder, but rather enhances, the anti-tumor effect of anti-CTLA-4 and anti-PD-1 combination treatment. To address potential clinical applicability, the authors further found the expression of TNF in checkpoint blockade-induced colitis to be increased in the colon when compared to healthy tissue. Based on these findings, they proposed a clinical application of TNF blockers in patients undergoing checkpoint blockade. In fact, a phase I clinical trial (https://clinicaltrials.gov; NCT03293784) is currently assessing the safety and impact on efficacy of the proposed approach. In line with the idea to optimize the "on target" aspect of checkpoint blockade, Zhang et al. designed and modified the $\mathrm{pH}$-sensitivity of an anti-CTLA-4 antibody. ${ }^{258}$ Because of the acidic 
$\mathrm{pH}$ in the TME, one would expect the anti-CTLA-4 antibody to be preferentially active in the acidic TME. With this approach the authors were able to prevent antibody-triggered lysosomal degradation. This led to an improved immunotherapeutic effect and decreased the occurrence of irAEs in tumor-bearing mice. ${ }^{258}$ However, such approaches would not be effective, if the main activity of the checkpoint blockade occurs in the draining lymph nodes and not in the TME. In the future, it will be important to identify additional agents and modifications that specially inhibit the checkpoint blockade-induced autoimmunity while not impeding the anti-tumor activity.

\section{CONCLUSION}

Immune checkpoint blockade recently transformed cancer treatment by showing remarkable efficacy in multiple types of cancer. However, treatment with immune checkpoint blockade is accompanied by severe autoimmune disease-like side effects that strongly limit the applicability of these drugs. As immune checkpoint blockade becomes more broadly used in cancer treatment, our understanding and treatment of these autoimmune-like irAEs need to improve. Based on our knowledge of the important function of co-inhibitory receptors in autoimmunity, immune checkpoint blockade could be improved using the basic knowledge of tissue-specific autoimmune responses and the ability of various molecules to operate in different effector $T$ cells. Unbiased genomic approaches should be used to define tumor-specific targets for immunotherapy. Additionally, comparisons of $T$ cells infiltrating tumors with those associated with autoimmune tissue destruction will allow identification and characterization of targets that may promote anti-tumor immunity but not drive autoimmune-like side effects. Also noteworthy is a growing body of research focused on novel tumor-restricted immunotherapy strategies beyond the scope of this review. These promising approaches include: (i) Vaccines targeting neoantigens $^{259-261}$; (ii) Chimeric antigen receptor (CAR) T-cell therapy ${ }^{262}$; (iii) Improved delivery of checkpoint blockade antibodies within the TME; (iv) Bispecific antibodies ${ }^{263-265}$; and (v) Molecular shields restricting local activity of checkpoint inhibitors. ${ }^{266}$

For future research, we propose studies designed to identify novel regulatory molecules that, when targeted, simultaneously enhance anti-tumor immunity yet suppress autoimmunity.

\section{ACKNOWLEDGEMENTS}

We are grateful for Mary Collins and Alina Bollhagen for critical reading of the review. We apologize to the authors of the publications that could not be cited in the scope of this review. A.S. was supported by a German Academic Scholarship Foundation (Studienstiftung des Deutschen Volkes) PhD fellowship. Work in our laboratory on this topic is supported by NIH grants 5P01AI073748, 5R01NS03084, 4R01NS045937, 5P01Al039671, and 5P01Al05629.

\section{AUTHOR CONTRIBUTIONS}

A.S., L.B. and V.K.K. wrote the manuscript. A.M. provided material for the figures and discussed the contents.

\section{ADDITIONAL INFORMATION}

Competing interests The authors declare no competing interests.

\section{REFERENCES}

1. Anderson, M. S. et al. Projection of an immunological self shadow within the thymus by the aire protein. Science 298, 1395-1401 (2002).

2. Derbinski, J., Schulte, A., Kyewski, B. \& Klein, L. Promiscuous gene expression in medullary thymic epithelial cells mirrors the peripheral self. Nat. Immunol. 2, 1032-1039 (2001).
3. Richards, D. M., Kyewski, B. \& Feuerer, M. Re-examining the nature and function of self-reactive T cells. Trends Immunol. 37, 114-125 (2016).

4. Mueller, D. L., Jenkins, M. K. \& Schwartz, R. H. Clonal expansion versus functional clonal inactivation: a costimulatory signalling pathway determines the outcome of T cell antigen receptor occupancy. Annu. Rev. Immunol. 7, 445-480 (1989).

5. Bretscher, P. \& Cohn, M. A theory of self-nonself discrimination. Science 169, 1042-1049 (1970).

6. Chen, L. \& Flies, D. B. Molecular mechanisms of T cell co-stimulation and coinhibition. Nat. Rev. Immunol. 13, 227-242 (2013).

7. June, C. H., Ledbetter, J. A., Gillespie, M. M., Lindsten, T. \& Thompson, C. B. T-cell proliferation involving the CD28 pathway is associated with cyclosporineresistant interleukin 2 gene expression. Mol. Cell Biol. 7, 4472-4481 (1987).

8. Francisco, L. M. et al. PD-L1 regulates the development, maintenance, and function of induced regulatory T cells. J. Exp. Med. 206, 3015-3029 (2009).

9. Wing, K. et al. CTLA-4 control over Foxp3+ regulatory $T$ cell function. Science 322, 271-275 (2008).

10. Zajac, A. J. et al. Viral immune evasion due to persistence of activated T cells without effector function. J. Exp. Med. 188, 2205-2213 (1998).

11. Wherry, E. J., Blattman, J. N., Murali-Krishna, K., van der Most, R. \& Ahmed, R. Viral persistence alters CD8 T-cell immunodominance and tissue distribution and results in distinct stages of functional impairment. J. Virol. 77, 4911-4927 (2003).

12. Wherry, E. J. \& Ahmed, R. Memory CD8 T-cell differentiation during viral infection. J. Virol. 78, 5535-5545 (2004).

13. Wherry, E. J. T cell exhaustion. Nat. Immunol. 12, 492-499 (2011).

14. Delpoux, A. et al. Continuous activity of Foxo1 is required to prevent anergy and maintain the memory state of CD8(+) T cells. J. Exp. Med. 215, 575-594 (2018).

15. Lang, K. S. et al. Inverse correlation between IL-7 receptor expression and CD8 T cell exhaustion during persistent antigen stimulation. Eur. J. Immunol. 35, 738-745 (2005).

16. Wherry, E. J., Barber, D. L., Kaech, S. M., Blattman, J. N. \& Ahmed, R. Antigenindependent memory CD8 T cells do not develop during chronic viral infection. Proc. Natl. Acad. Sci. USA 101, 16004-16009 (2004).

17. Oxenius, A., Zinkernagel, R. M. \& Hengartner, H. Comparison of activation versus induction of unresponsiveness of virus-specific CD4+ and CD8+ T cells upon acute versus persistent viral infection. Immunity 9, 449-457 (1998).

18. Antoine, $\mathrm{P}$. et al. Functional exhaustion of $\mathrm{CD} 4+\mathrm{T}$ lymphocytes during primary cytomegalovirus infection. J. Immunol. 189, 2665-2672 (2012).

19. Chen, C. Y. et al. Combined treatment with HMGN1 and anti-CD4 depleting antibody reverses $\mathrm{T}$ cell exhaustion and exerts robust anti-tumor effects in mice. J. Immunother. Cancer 7, 21 (2019).

20. Penaloza-MacMaster, P., Provine, N. M., Blass, E. \& Barouch, D. H. CD4 T cell depletion substantially augments the rescue potential of PD-L1 blockade for deeply exhausted CD8 T cells. J. Immunol. 195, 1054-1063 (2015).

21. Sen, D. R. et al. The epigenetic landscape of T cell exhaustion. Science 354, 1165-1169 (2016).

22. Wherry, E. J. et al. Molecular signature of CD8+ T cell exhaustion during chronic viral infection. Immunity 27, 670-684 (2007).

23. Barber, D. L. et al. Restoring function in exhausted CD8 T cells during chronic viral infection. Nature 439, 682-687 (2006).

24. Freeman, G. J. et al. Engagement of the PD-1 immunoinhibitory receptor by a novel B7 family member leads to negative regulation of lymphocyte activation. J. Exp. Med. 192, 1027-1034 (2000).

25. Brown, J. A. et al. Blockade of programmed death-1 ligands on dendritic cells enhances T cell activation and cytokine production. J. Immunol. 170, 1257-1266 (2003).

26. Velu, V. et al. Enhancing SIV-specific immunity in vivo by PD-1 blockade. Nature 458, 206-210 (2009).

27. Quigley, M. et al. Transcriptional analysis of HIV-specific CD8+ T cells shows that PD-1 inhibits T cell function by upregulating BATF. Nat. Med. 16, 1147-1151 (2010).

28. Bowen, D. G. \& Walker, C. M. Adaptive immune responses in acute and chronic hepatitis C virus infection. Nature 436, 946-952 (2005).

29. Ye, B. et al. T-cell exhaustion in chronic hepatitis B infection: current knowledge and clinical significance. Cell Death Dis. 6, e1694 (2015).

30. Baitsch, L. et al. Exhaustion of tumor-specific CD8(+) T cells in metastases from melanoma patients. J. Clin. Invest. 121, 2350-2360 (2011).

31. McKinney, E. F., Lee, J. C., Jayne, D. R., Lyons, P. A. \& Smith, K. G. T-cell exhaustion, co-stimulation and clinical outcome in autoimmunity and infection. Nature 523, 612-616 (2015).

32. Dardalhon, V., Korn, T., Kuchroo, V. K. \& Anderson, A. C. Role of Th1 and Th17 cells in organ-specific autoimmunity. J. Autoimmun. 31, 252-256 (2008).

33. Ben-Nun, A. \& Cohen, I. R. Vaccination against autoimmune encephalomyelitis (EAE): attenuated autoimmune T lymphocytes confer resistance to induction of 
active EAE but not to EAE mediated by the intact T lymphocyte line. Eur. J. Immunol. 11, 949-952 (1981).

34. Pettinelli, C. B. \& McFarlin, D. E. Adoptive transfer of experimental allergic encephalomyelitis in SJL/J mice after in vitro activation of lymph node cells by myelin basic protein: requirement for Lyt 1+ 2- T lymphocytes. J. Immunol. 127, 1420-1423 (1981).

35. Renno, T., Krakowski, M., Piccirillo, C., Lin, J. Y. \& Owens, T. TNF-alpha expression by resident microglia and infiltrating leukocytes in the central nervous system of mice with experimental allergic encephalomyelitis. Regulation by Th1 cytokines. J. Immunol. 154, 944-953 (1995).

36. Bettelli, E. et al. Loss of T-bet, but not STAT1, prevents the development of experimental autoimmune encephalomyelitis. J. Exp. Med. 200, 79-87 (2004).

37. Chitnis, T. et al. Effect of targeted disruption of STAT4 and STAT6 on the induction of experimental autoimmune encephalomyelitis. J. Clin. Invest. 108, 739-747 (2001).

38. Germann, T., Hess, H., Szeliga, J. \& Rude, E. Characterization of the adjuvant effect of IL-12 and efficacy of IL-12 inhibitors in type II collagen-induced arthritis. Ann. N. Y. Acad. Sci. 795, 227-240 (1996).

39. Krakowski, M. \& Owens, T. Interferon-gamma confers resistance to experimental allergic encephalomyelitis. Eur. J. Immunol. 26, 1641-1646 (1996).

40. Willenborg, D. O., Fordham, S., Bernard, C. C., Cowden, W. B. \& Ramshaw, I. A IFN-gamma plays a critical down-regulatory role in the induction and effector phase of myelin oligodendrocyte glycoprotein-induced autoimmune encephalomyelitis. J. Immunol. 157, 3223-3227 (1996).

41. Lock, C. et al. Gene-microarray analysis of multiple sclerosis lesions yields new targets validated in autoimmune encephalomyelitis. Nat. Med. 8, 500-508 (2002).

42. Matusevicius, D. et al. Interleukin-17 mRNA expression in blood and CSF mononuclear cells is augmented in multiple sclerosis. Mult. Scler. 5, 101-104 (1999).

43. Aarvak, T., Chabaud, M., Miossec, P. \& Natvig, J. B. IL-17 is produced by some proinflammatory Th1/Th0 cells but not by Th2 cells. J. Immunol. 162, 1246-1251 (1999).

44. Teunissen, M. B., Koomen, C. W., de Waal Malefyt, R., Wierenga, E. A. \& Bos, J. D. Interleukin-17 and interferon-gamma synergize in the enhancement of proinflammatory cytokine production by human keratinocytes. J. Invest. Dermatol. 111, 645-649 (1998).

45. Burkett, P. R., Meyer zu Horste, G. \& Kuchroo, V. K. Pouring fuel on the fire: Th17 cells, the environment, and autoimmunity. J. Clin. Invest. 125, 2211-2219 (2015).

46. Bettelli, E. et al. Reciprocal developmental pathways for the generation of pathogenic effector TH17 and regulatory T cells. Nature 441, 235-238 (2006).

47. Sutton, C., Brereton, C., Keogh, B., Mills, K. H. \& Lavelle, E. C. A crucial role for interleukin (IL)-1 in the induction of IL-17-producing $T$ cells that mediate autoimmune encephalomyelitis. J. Exp. Med. 203, 1685-1691 (2006).

48. Langrish, C. L. et al. IL-23 drives a pathogenic T cell population that induces autoimmune inflammation. J. Exp. Med. 201, 233-240 (2005).

49. Levesque, S. A. et al. Myeloid cell transmigration across the CNS vasculature triggers IL-1beta-driven neuroinflammation during autoimmune encephalomyelitis in mice. J. Exp. Med. 213, 929-949 (2016).

50. Okuda, Y. et al. IL-6 plays a crucial role in the induction phase of myelin oligodendrocyte glucoprotein 35-55 induced experimental autoimmune encephalomyelitis. J. Neuroimmunol. 101, 188-196 (1999).

51. Thakker, P. et al. IL-23 is critical in the induction but not in the effector phase of experimental autoimmune encephalomyelitis. J. Immunol. 178, 2589-2598 (2007).

52. Korn, T. et al. Myelin-specific regulatory T cells accumulate in the CNS but fail to control autoimmune inflammation. Nat. Med. 13, 423-431 (2007).

53. Gaublomme, J. T. et al. Single-cell genomics unveils critical regulators of Th17 cell pathogenicity. Cell 163, 1400-1412 (2015).

54. Hirota, K. et al. Fate mapping of IL-17-producing $\mathrm{T}$ cells in inflammatory responses. Nat. Immunol. 12, 255-263 (2011).

55. Meyer Zu Horste, G. et al. Fas promotes T Helper 17 cell differentiation and inhibits $T$ helper 1 cell development by binding and sequestering transcription factor STAT1. Immunity 48, 556-569 e557 (2018).

56. Burkett, P. R. \& Kuchroo, V. K. IL-17 blockade in psoriasis. Cell 167, 1669 (2016).

57. Chatzantoni, K. \& Mouzaki, A. Anti-TNF-alpha antibody therapies in autoimmune diseases. Curr. Top. Med. Chem. 6, 1707-1714 (2006).

58. June, C. H., Warshauer, J. T. \& Bluestone, J. A. Is autoimmunity the Achilles' heel of cancer immunotherapy? Nat. Med. 23, 540-547 (2017).

59. Michot, J. M. et al. Immune-related adverse events with immune checkpoint blockade: a comprehensive review. Eur. J. Cancer 54, 139-148 (2016).

60. Brunet, J. F. et al. A new member of the immunoglobulin superfamily-CTLA-4. Nature 328, 267-270 (1987).

61. Linsley, P. S. et al. CTLA-4 is a second receptor for the B cell activation antigen B7. J. Exp. Med. 174, 561-569 (1991).
62. Walunas, T. L. et al. CTLA-4 can function as a negative regulator of T cell activation. Immunity 1, 405-413 (1994).

63. Lenschow, D. J., Walunas, T. L. \& Bluestone, J. A. CD28/B7 system of T cell costimulation. Annu. Rev. Immunol. 14, 233-258 (1996).

64. Linsley, P. S. et al. Human B7-1 (CD80) and B7-2 (CD86) bind with similar avidities but distinct kinetics to CD28 and CTLA-4 receptors. Immunity 1, 793-801 (1994).

65. Krummel, M. F., Sullivan, T. J. \& Allison, J. P. Superantigen responses and costimulation: CD28 and CTLA-4 have opposing effects on T cell expansion in vitro and in vivo. Int. Immunol. 8, 519-523 (1996).

66. Leach, D. R., Krummel, M. F. \& Allison, J. P. Enhancement of antitumor immunity by CTLA-4 blockade. Science 271, 1734-1736 (1996).

67. Tivol, E. A. et al. Loss of CTLA-4 leads to massive lymphoproliferation and fatal multiorgan tissue destruction, revealing a critical negative regulatory role of CTLA-4. Immunity 3, 541-547 (1995).

68. Waterhouse, P. et al. Lymphoproliferative disorders with early lethality in mice deficient in Ctla-4. Science 270, 985-988 (1995).

69. Klocke, K., Sakaguchi, S., Holmdahl, R. \& Wing, K. Induction of autoimmune disease by deletion of CTLA-4 in mice in adulthood. Proc. Natl. Acad. Sci. USA 113, E2383-E2392 (2016).

70. Kristiansen, O. P., Larsen, Z. M. \& Pociot, F. CTLA-4 in autoimmune diseases-a general susceptibility gene to autoimmunity? Genes Immun. 1, 170-184 (2000).

71. Luhder, F., Hoglund, P., Allison, J. P., Benoist, C. \& Mathis, D. Cytotoxic T lymphocyte-associated antigen 4 (CTLA-4) regulates the unfolding of autoimmune diabetes. J. Exp. Med. 187, 427-432 (1998).

72. Karandikar, N. J., Vanderlugt, C. L., Walunas, T. L., Miller, S. D. \& Bluestone, J. A CTLA-4: a negative regulator of autoimmune disease. J. Exp. Med. 184, 783-788 (1996).

73. Paterson, A. M. et al. Deletion of CTLA-4 on regulatory T cells during adulthood leads to resistance to autoimmunity. J. Exp. Med. 212, 1603-1621 (2015).

74. Magistrelli, G. et al. A soluble form of CTLA-4 generated by alternative splicing is expressed by nonstimulated human T cells. Eur. J. Immunol. 29, 3596-3602 (1999).

75. Ueda, H. et al. Association of the T-cell regulatory gene CTLA4 with susceptibility to autoimmune disease. Nature 423, 506-511 (2003).

76. Araki, M. et al. Genetic evidence that the differential expression of the ligandindependent isoform of CTLA-4 is the molecular basis of the Idd5.1 type 1 diabetes region in nonobese diabetic mice. J. Immunol. 183, 5146-5157 (2009).

77. Stumpf, M., Zhou, X. \& Bluestone, J. A. The B7-independent isoform of CTLA-4 functions to regulate autoimmune diabetes. J. Immunol. 190, 961-969 (2013).

78. Vijayakrishnan, L. et al. An autoimmune disease-associated CTLA-4 splice variant lacking the B7 binding domain signals negatively in T cells. Immunity 20, 563-575 (2004).

79. Ishida, Y., Agata, Y., Shibahara, K. \& Honjo, T. Induced expression of PD-1, a novel member of the immunoglobulin gene superfamily, upon programmed cell death. EMBO J. 11, 3887-3895 (1992).

80. Francisco, L. M., Sage, P. T. \& Sharpe, A. H. The PD-1 pathway in tolerance and autoimmunity. Immunol. Rev. 236, 219-242 (2010).

81. Nishimura, H. et al. Autoimmune dilated cardiomyopathy in PD-1 receptordeficient mice. Science 291, 319-322 (2001).

82. Nishimura, H., Nose, M., Hiai, H., Minato, N. \& Honjo, T. Development of lupuslike autoimmune diseases by disruption of the PD-1 gene encoding an ITIM motif-carrying immunoreceptor. Immunity 11, 141-151 (1999).

83. Wang, J. et al. Establishment of NOD-Pdcd1 ${ }^{-1-}$ mice as an efficient animal model of type I diabetes. Proc. Natl. Acad. Sci. USA 102, 11823-11828 (2005).

84. Salama, A. D. et al. Critical role of the programmed death-1 (PD-1) pathway in regulation of experimental autoimmune encephalomyelitis. J. Exp. Med. 198, 71-78 (2003).

85. Prokunina, L. et al. A regulatory polymorphism in PDCD1 is associated with susceptibility to systemic lupus erythematosus in humans. Nat. Genet. 32, 666-669 (2002).

86. Nielsen, C., Hansen, D., Husby, S., Jacobsen, B. B. \& Lillevang, S. T. Association of a putative regulatory polymorphism in the PD-1 gene with susceptibility to type 1 diabetes. Tissue Antigens 62, 492-497 (2003).

87. Lee, S. H. et al. Association of the programmed cell death 1 (PDCD1) gene polymorphism with ankylosing spondylitis in the Korean population. Arthritis Res. Ther. 8, R163 (2006).

88. Prokunina, L. et al. Association of the PD-1.3A allele of the PDCD1 gene in patients with rheumatoid arthritis negative for rheumatoid factor and the shared epitope. Arthritis Rheum. 50, 1770-1773 (2004).

89. Monney, L. et al. Th1-specific cell surface protein Tim-3 regulates macrophage activation and severity of an autoimmune disease. Nature 415, 536-541 (2002).

90. Anderson, A. C., Joller, N. \& Kuchroo, V. K. Lag-3, Tim-3, and TIGIT: co-inhibitory receptors with specialized functions in immune regulation. Immunity $\mathbf{4 4}$, 989-1004 (2016). 
91. Zhu, C. et al. The Tim-3 ligand galectin-9 negatively regulates T helper type 1 immunity. Nat. Immunol. 6, 1245-1252 (2005).

92. Dekruyff, R. H. et al. T cell/transmembrane, Ig, and mucin-3 allelic variants differentially recognize phosphatidylserine and mediate phagocytosis of apoptotic cells. J. Immunol. 184, 1918-1930 (2010).

93. Huang, Y. H. et al. CEACAM1 regulates TIM-3-mediated tolerance and exhaustion. Nature 517, 386-390 (2015).

94. $\mathrm{Li}$, X. et al. Involvement of T cell Ig Mucin-3 (Tim-3) in the negative regulation of inflammatory bowel disease. Clin. Immunol. 134, 169-177 (2010).

95. Sanchez-Fueyo, A. et al. Tim-3 inhibits T helper type 1-mediated auto- and alloimmune responses and promotes immunological tolerance. Nat. Immunol. 4, 1093-1101 (2003).

96. Koguchi, K. et al. Dysregulated T cell expression of TIM3 in multiple sclerosis. J. Exp. Med. 203, 1413-1418 (2006).

97. Boivin, N., Baillargeon, J., Doss, P. M., Roy, A. P. \& Rangachari, M. Interferon-beta suppresses murine Th1 cell function in the absence of antigen-presenting cells. PLOS ONE 10, e0124802 (2015).

98. Kanai, Y., Satoh, T., Igawa, K. \& Yokozeki, H. Impaired expression of Tim-3 on Th17 and Th1 cells in psoriasis. Acta Derm. Venereol. 92, 367-371 (2012).

99. Liu, Y. et al. Increased Tim-3 expression on peripheral lymphocytes from patients with rheumatoid arthritis negatively correlates with disease activity. Clin. Immunol. 137, 288-295 (2010).

100. Shi, F. et al. Dysregulated Tim-3 expression and its correlation with imbalanced CD4 helper T cell function in ulcerative colitis. Clin. Immunol. 145, 230-240 (2012).

101. Hastings, W. D. et al. TIM-3 is expressed on activated human CD4+ T cells and regulates Th1 and Th17 cytokines. Eur. J. Immunol. 39, 2492-2501 (2009).

102. Gayden, T. et al. Germline HAVCR2 mutations altering TIM-3 characterize subcutaneous panniculitis-like T cell lymphomas with hemophagocytic lymphohistiocytic syndrome. Nat. Genet. 50, 1650-1657 (2018).

103. Lee, J., Phong, B., Egloff, A. M. \& Kane, L. P. TIM polymorphisms-genetics and function. Genes Immun. 12, 595-604 (2011).

104. Boles, K. S. et al. A novel molecular interaction for the adhesion of follicular CD4 T cells to follicular DC. Eur. J. Immunol. 39, 695-703 (2009).

105. Joller, N. et al. Treg cells expressing the coinhibitory molecule TIGIT selectively inhibit proinflammatory Th1 and Th17 cell responses. Immunity 40, 569-581 (2014).

106. $\mathrm{Yu}, \mathrm{X}$. et al. The surface protein TIGIT suppresses T cell activation by promoting the generation of mature immunoregulatory dendritic cells. Nat. Immunol. 10, 48-57 (2009)

107. Stanietsky, N. et al. The interaction of TIGIT with PVR and PVRL2 inhibits human NK cell cytotoxicity. Proc. Natl. Acad. Sci. USA 106, 17858-17863 (2009).

108. Joller, N. et al. Cutting edge: TIGIT has T cell-intrinsic inhibitory functions. J. Immunol. 186, 1338-1342 (2011)

109. Levin, S. D. et al. Vstm3 is a member of the CD28 family and an important modulator of T-cell function. Eur. J. Immunol. 41, 902-915 (2011).

110. Hafler, J. P. et al. CD226 Gly307Ser association with multiple autoimmune diseases. Genes Immun. 10, 5-10 (2009).

111. Maiti, A. K. et al. Non-synonymous variant (Gly307Ser) in CD226 is associated with susceptibility to multiple autoimmune diseases. Rheumatol. (Oxf.) 49, 1239-1244 (2010).

112. Triebel, F. et al. LAG-3, a novel lymphocyte activation gene closely related to CD4. J. Exp. Med. 171, 1393-1405 (1990).

113. Huard, B., Prigent, P., Tournier, M., Bruniquel, D. \& Triebel, F. CD4/major histocompatibility complex class II interaction analyzed with CD4- and lymphocyte activation gene-3 (LAG-3)-Ig fusion proteins. Eur. J. Immunol. 25, 2718-2721 (1995).

114. Xu, F. et al. LSECtin expressed on melanoma cells promotes tumor progression by inhibiting antitumor T-cell responses. Cancer Res. 74, 3418-3428 (2014).

115. Wang, J. et al. Fibrinogen-like protein 1 is a major immune inhibitory ligand of LAG-3. Cell 176, 334-347 e312 (2019).

116. Jha, V. et al. Lymphocyte Activation Gene-3 (LAG-3) negatively regulates environmentally-induced autoimmunity. PLOS ONE 9, e104484 (2014).

117. Bettini, M. et al. Cutting edge: accelerated autoimmune diabetes in the absence of LAG-3. J. Immunol. 187, 3493-3498 (2011).

118. McKinney, E. F. \& Smith, K. G. T cell exhaustion and immune-mediated diseasethe potential for therapeutic exhaustion. Curr. Opin. Immunol. 43, 74-80 (2016).

119. Paluch, C., Santos, A. M., Anzilotti, C., Cornall, R. J. \& Davis, S. J. Immune checkpoints as therapeutic targets in autoimmunity. Front. Immunol. 9, 2306 (2018).

120. Dixon, K. O. et al. Functional anti-TIGIT antibodies regulate development of autoimmunity and antitumor immunity. J. Immunol. 200, 3000-3007 (2018).

121. Raptopoulou, A. P. et al. The programmed death 1 /programmed death ligand 1 inhibitory pathway is up-regulated in rheumatoid synovium and regulates peripheral T cell responses in human and murine arthritis. Arthritis Rheum. 62, 1870-1880 (2010).

122. Seki, M. et al. Galectin-9 suppresses the generation of Th17, promotes the induction of regulatory $T$ cells, and regulates experimental autoimmune arthritis. Clin. Immunol. 127, 78-88 (2008).

123. Chihara, N. et al. Induction and transcriptional regulation of the co-inhibitory gene module in T cells. Nature 558, 454-459 (2018).

124. Delaney, C. et al. Combinatorial prediction of marker panels from single-cell transcriptomic data. Mol. Syst. Biol. 15, e9005 (2019).

125. Ehrlich, P. Beitr@pge zur experimentellen Pathologie und Chemotherapie. (Akademische Verlagsgesellschaft, 1909).

126. Coley, W. B. II Contribution to the knowledge of sarcoma. Ann. Surg. 14, 199-220 (1891).

127. Coley, W. B. The treatment of inoperable sarcoma by bacterial toxins (the mixed toxins of the streptococcus erysipelas and the Bacillus prodigiosus). Proc. R. Soc. Med. 3, 1-48 (1910).

128. Burnet, M. Immunological factors in the process of carcinogenesis. Br. Med. Bull. 20, 154-158 (1964)

129. Burnet, F. M. The concept of immunological surveillance. Prog. Exp. Tumor Res. 13, 1-27 (1970).

130. Alexandrov, L. B. et al. Signatures of mutational processes in human cancer. Nature 500, 415-421 (2013).

131. Koebel, C. M. et al. Adaptive immunity maintains occult cancer in an equilibrium state. Nature 450, 903-907 (2007).

132. Shankaran, V. et al. IFNgamma and lymphocytes prevent primary tumour development and shape tumour immunogenicity. Nature 410, 1107-1111 (2001).

133. Castle, J. C. et al. Exploiting the mutanome for tumor vaccination. Cancer Res. $\mathbf{7 2}$, 1081-1091 (2012).

134. Matsushita, H. et al. Cancer exome analysis reveals a T-cell-dependent mechanism of cancer immunoediting. Nature 482, 400-404 (2012).

135. Boon, T. \& Old, L. J. Cancer tumor antigens. Curr. Opin. Immunol. 9, 681-683 (1997).

136. Ikeda, H., Old, L. J. \& Schreiber, R. D. The roles of IFN gamma in protection against tumor development and cancer immunoediting. Cytokine Growth Factor Rev. 13, 95-109 (2002).

137. Wherry, E. J. et al. Lineage relationship and protective immunity of memory CD8 T cell subsets. Nat. Immunol. 4, 225-234 (2003).

138. Kaech, S. M. \& Cui, W. Transcriptional control of effector and memory CD8+ T cell differentiation. Nat. Rev. Immunol. 12, 749-761 (2012).

139. Sallusto, F., Lenig, D., Forster, R., Lipp, M. \& Lanzavecchia, A. Two subsets of memory $\mathrm{T}$ lymphocytes with distinct homing potentials and effector functions. Nature 401, 708-712 (1999).

140. Fridman, W. H., Pages, F., Sautes-Fridman, C. \& Galon, J. The immune contexture in human tumours: impact on clinical outcome. Nat. Rev. Cancer 12, 298-306 (2012).

141. Pages, F. et al. International validation of the consensus Immunoscore for the classification of colon cancer: a prognostic and accuracy study. Lancet 391, 2128-2139 (2018).

142. Moskophidis, D., Lechner, F., Pircher, H. \& Zinkernagel, R. M. Virus persistence in acutely infected immunocompetent mice by exhaustion of antiviral cytotoxic effector T cells. Nature 362, 758-761 (1993).

143. Lee, P. P. et al. Characterization of circulating T cells specific for tumorassociated antigens in melanoma patients. Nat. Med. 5, 677-685 (1999).

144. Zippelius, A. et al. Effector function of human tumor-specific CD8 T cells in melanoma lesions: a state of local functional tolerance. Cancer Res. 64, 2865-2873 (2004).

145. Singer, $M$. et al. A distinct gene module for dysfunction uncoupled from activation in tumor-infiltrating T cells. Cell 166, 1500-1511 e1509 (2016).

146. Vodnala, S. K. et al. T cell stemness and dysfunction in tumors are triggered by a common mechanism. Science 363, https://doi.org/10.1126/science.aau0135 (2019).

147. Alfei, F. et al. TOX reinforces the phenotype and longevity of exhausted T cells in chronic viral infection. Nature 571, 265-269 (2019).

148. Scott, A. C. et al. TOX is a critical regulator of tumour-specific T cell differentiation. Nature 571, 270-274 (2019).

149. Khan, O. et al. TOX transcriptionally and epigenetically programs CD8(+) T cell exhaustion. Nature 571, 211-218 (2019).

150. Chambers, C. A. \& Allison, J. P. CTLA-4-the costimulatory molecule that doesn't: regulation of T-cell responses by inhibition. Cold Spring Harb. Symp. Quant. Biol. 64, 303-312 (1999).

151. Greenfield, E. A. et al. B7.2 expressed by T cells does not induce CD28-mediated costimulatory activity but retains CTLA4 binding: implications for induction of antitumor immunity to T cell tumors. J. Immunol. 158, 2025-2034 (1997). 
152. Contardi, E. et al. CTLA-4 is constitutively expressed on tumor cells and can trigger apoptosis upon ligand interaction. Int. J. Cancer 117, 538-550 (2005).

153. Paradis, T. J. et al. The anti-tumor activity of anti-CTLA-4 is mediated through its induction of IFN gamma. Cancer Immunol. Immunother. 50, 125-133 (2001).

154. Shrikant, P., Khoruts, A. \& Mescher, M. F. CTLA-4 blockade reverses CD8+ T cell tolerance to tumor by a CD4+ T cell- and IL-2-dependent mechanism. Immunity 11, 483-493 (1999).

155. Sotomayor, E. M., Borrello, I., Tubb, E., Allison, J. P. \& Levitsky, H. I. In vivo blockade of CTLA-4 enhances the priming of responsive T cells but fails to prevent the induction of tumor antigen-specific tolerance. Proc. Natl. Acad. Sci. USA 96, 11476-11481 (1999).

156. Simpson, T. R. et al. Fc-dependent depletion of tumor-infiltrating regulatory $T$ cells co-defines the efficacy of anti-CTLA-4 therapy against melanoma. J. Exp. Med. 210, 1695-1710 (2013).

157. Roncella, S. et al. CTLA-4 in mesothelioma patients: tissue expression, body fluid levels and possible relevance as a prognostic factor. Cancer Immunol. Immunother. 65, 909-917 (2016).

158. Huang, P. Y. et al. Tumor CTLA-4 overexpression predicts poor survival in patients with nasopharyngeal carcinoma. Oncotarget 7, 13060-13068 (2016).

159. Shah, K. V., Chien, A. J., Yee, C. \& Moon, R. T. CTLA-4 is a direct target of Wnt/ beta-catenin signaling and is expressed in human melanoma tumors. J. Invest. Dermatol. 128, 2870-2879 (2008).

160. Paulsen, E. E. et al. CTLA-4 expression in the non-small cell lung cancer patient tumor microenvironment: diverging prognostic impact in primary tumors and lymph node metastases. Cancer Immunol. Immunother. 66, 1449-1461 (2017).

161. Sun, T., Hu, Z., Shen, H. \& Lin, D. Genetic polymorphisms in cytotoxic Tlymphocyte antigen 4 and cancer: the dialectical nature of subtle human immune dysregulation. Cancer Res. 69, 6011-6014 (2009).

162. Yang, M. et al. The functional cytotoxic T lymphocyte-associated Protein 4 49Gto-A genetic variant and risk of pancreatic cancer. Cancer 118, 4681-4686 (2012).

163. Li, D. et al. Association of CTLA-4 gene polymorphisms with sporadic breast cancer risk and clinical features in Han women of northeast China. Mol. Cell Biochem. 364, 283-290 (2012).

164. Latchman, Y. et al. PD-L2 is a second ligand for PD-1 and inhibits T cell activation. Nat. Immunol. 2, 261-268 (2001).

165. Ishida, M. et al. Differential expression of PD-L1 and PD-L2, ligands for an inhibitory receptor PD-1, in the cells of lymphohematopoietic tissues. Immunol. Lett. 84, 57-62 (2002)

166. Iwai, Y. et al. Involvement of PD-L1 on tumor cells in the escape from host immune system and tumor immunotherapy by PD-L1 blockade. Proc. Natl. Acad. Sci. USA 99, 12293-12297 (2002)

167. Juneja, V. R. et al. PD-L1 on tumor cells is sufficient for immune evasion in immunogenic tumors and inhibits CD8 T cell cytotoxicity. J. Exp. Med. 214, 895-904 (2017).

168. Curiel, T. J. et al. Blockade of B7-H1 improves myeloid dendritic cell-mediated antitumor immunity. Nat. Med. 9, 562-567 (2003)

169. Strome, S. E. et al. B7-H1 blockade augments adoptive T-cell immunotherapy for squamous cell carcinoma. Cancer Res. 63, 6501-6505 (2003)

170. Blank, C. et al. PD-L1/B7H-1 inhibits the effector phase of tumor rejection by $T$ cell receptor (TCR) transgenic CD8+ T cells. Cancer Res. 64, 1140-1145 (2004).

171. He, Y. F. et al. Blocking programmed death-1 ligand-PD-1 interactions by local gene therapy results in enhancement of antitumor effect of secondary lymphoid tissue chemokine. J. Immunol. 173, 4919-4928 (2004).

172. Chen, J., Jiang, C. C., Jin, L. \& Zhang, X. D. Regulation of PD-L1: a novel role of pro-survival signalling in cancer. Ann. Oncol. 27, 409-416 (2016).

173. Khunger, $M$. et al. Programmed cell death 1 (PD-1) ligand (PD-L1) expression in solid tumors as a predictive biomarker of benefit from PD-1/PD-L1 axis inhibitors: a systematic review and meta-analysis. JCO Precis. Oncol., 1-15, https://doi. org/10.1200/po.16.00030 (2017).

174. Salmaninejad, A. et al. PD-1 and cancer: molecular mechanisms and polymorphisms. Immunogenetics 70, 73-86 (2018).

175. Dardalhon, V. et al. Tim-3/galectin-9 pathway: regulation of Th1 immunity through promotion of $\mathrm{CD} 11 \mathrm{~b}+\mathrm{Ly}-6 \mathrm{G}+$ myeloid cells. J. Immunol. 185, 1383-1392 (2010).

176. Anderson, A. C. et al. T-bet, a Th1 transcription factor regulates the expression of Tim-3. Eur. J. Immunol. 40, 859-866 (2010).

177. Sabatos, C. A. et al. Interaction of Tim-3 and Tim-3 ligand regulates T helper type 1 responses and induction of peripheral tolerance. Nat. Immunol. 4, 1102-1110 (2003).

178. Fourcade, J. et al. PD-1 and Tim-3 regulate the expansion of tumor antigenspecific $\mathrm{CD} 8(+) \mathrm{T}$ cells induced by melanoma vaccines. Cancer Res. 74, 1045-1055 (2014)
179. Sakuishi, K. et al. Targeting Tim-3 and PD-1 pathways to reverse $T$ cell exhaustion and restore anti-tumor immunity. J. Exp. Med. 207, 2187-2194 (2010).

180. Rangachari, M. et al. Bat3 promotes $T$ cell responses and autoimmunity by repressing Tim-3-mediated cell death and exhaustion. Nat. Med. 18, 1394-1400 (2012).

181. Zhang, Y., Cai, P., Liang, T., Wang, L. \& Hu, L. TIM-3 is a potential prognostic marker for patients with solid tumors: a systematic review and meta-analysis. Oncotarget 8, 31705-31713 (2017).

182. Xu, B. et al. Circulating and tumor-infiltrating Tim-3 in patients with colorectal cancer. Oncotarget 6, 20592-20603 (2015).

183. Cai, C. et al. T-cell immunoglobulin- and mucin-domain-containing molecule 3 gene polymorphisms and renal cell carcinoma. DNA Cell Biol. 31, 1285-1289 (2012).

184. Tong, D. et al. T cell immunoglobulin- and mucin-domain-containing molecule 3 gene polymorphisms and susceptibility to pancreatic cancer. Mol. Biol. Rep. 39, 9941-9946 (2012)

185. Bai, J. et al. T-cell immunoglobulin- and mucin-domain-containing molecule 3 gene polymorphisms and prognosis of non-small-cell lung cancer. Tumour Biol. 34, 805-809 (2013).

186. Gao, X., Yang, J., He, Y. \& Zhang, J. Quantitative assessment of TIM-3 polymorphisms and cancer risk in Chinese Han population. Oncotarget 7, 35768-35775 (2016).

187. He, Y. et al. TIM-3, a promising target for cancer immunotherapy. Onco Targets Ther. 11, 7005-7009 (2018).

188. Johnston, R. J. et al. The immunoreceptor TIGIT regulates antitumor and antiviral CD8(+) T cell effector function. Cancer Cell 26, 923-937 (2014).

189. Chauvin, J. M. et al. TIGIT and PD-1 impair tumor antigen-specific CD8(+) T cells in melanoma patients. J. Clin. Invest. 125, 2046-2058 (2015).

190. Inozume, T. et al. Melanoma cells control anti-melanoma CTL responses via interaction between TIGIT and CD155 in the effector phase. J. Invest. Dermatol. https://doi.org/10.1038/jid.2015.404 (2015).

191. Blessin, N. C. et al. Patterns of TIGIT expression in lymphatic tissue, inflammation, and cancer. Dis. Markers 2019, 5160565 (2019).

192. Li, X. Y. et al. CD155 loss enhances tumor suppression via combined host and tumor-intrinsic mechanisms. J. Clin. Invest. 128, 2613-2625 (2018).

193. Zhang, B. et al. Immunoreceptor TIGIT inhibits the cytotoxicity of human cytokine-induced killer cells by interacting with CD155. Cancer Immunol. Immunother. 65, 305-314 (2016).

194. Mahnke, K. \& Enk, A. H. TIGIT-CD155 interactions in melanoma: a novel coinhibitory pathway with potential for clinical intervention. J. Invest. Dermatol. 136, 9-11 (2016).

195. Kurtulus, S. et al. TIGIT predominantly regulates the immune response via regulatory T cells. J. Clin. Invest. 125, 4053-4062 (2015).

196. Hung, A. L. et al. TIGIT and PD-1 dual checkpoint blockade enhances antitumor immunity and survival in GBM. Oncoimmunology 7, e1466769 (2018).

197. Josefsson, S. E. et al. TIGIT and PD-1 mark intratumoral T cells with reduced effector function in B-cell non-hodgkin lymphoma. Cancer Immunol. Res. 7, 355-362 (2019).

198. Fourcade, J. et al. CD226 opposes TIGIT to disrupt Tregs in melanoma. JCI Insight 3, https://doi.org/10.1172/jci.insight.121157 (2018).

199. Catakovic, K. et al. TIGIT expressing CD4+T cells represent a tumor-supportive T cell subset in chronic lymphocytic leukemia. Oncoimmunology 7, e1371399 (2017).

200. Kong, Y. et al. T-cell immunoglobulin and ITIM domain (TIGIT) associates with CD8+ T-cell exhaustion and poor clinical outcome in AML patients. Clin. Cancer Res. 22, 3057-3066 (2016).

201. Solomon, B. L. \& Garrido-Laguna, I. TIGIT: a novel immunotherapy target moving from bench to bedside. Cancer Immunol. Immunother. 67, 1659-1667 (2018).

202. Wu, L. et al. Blockade of TIGIT/CD155 signaling reverses T-cell exhaustion and enhances antitumor capability in head and neck squamous cell carcinoma. Cancer Immunol. Res. https://doi.org/10.1158/2326-6066.CIR-18-0725 (2019).

203. Grosso, J. F. et al. LAG-3 regulates CD8+ T cell accumulation and effector function in murine self- and tumor-tolerance systems. J. Clin. Invest. 117, 3383-3392 (2007)

204. Gandhi, M. K. et al. Expression of LAG-3 by tumor-infiltrating lymphocytes is coincident with the suppression of latent membrane antigen-specific CD8+ Tcell function in Hodgkin lymphoma patients. Blood 108, 2280-2289 (2006).

205. Demeure, C. E., Wolfers, J., Martin-Garcia, N., Gaulard, P. \& Triebel, F. T Lymphocytes infiltrating various tumour types express the MHC class II ligand lymphocyte activation gene-3 (LAG-3): role of LAG-3/MHC class II interactions in cell-cell contacts. Eur. J. Cancer 37, 1709-1718 (2001).

206. Casati, C. et al. Soluble human LAG-3 molecule amplifies the in vitro generation of type 1 tumor-specific immunity. Cancer Res. 66, 4450-4460 (2006). 
207. Huard, B., Tournier, M. \& Triebel, F. LAG-3 does not define a specific mode of natural killing in human. Immunol. Lett. 61, 109-112 (1998).

208. Miyazaki, T., Dierich, A., Benoist, C. \& Mathis, D. Independent modes of natural killing distinguished in mice lacking Lag3. Science 272, 405-408 (1996).

209. Wierz, M. et al. Dual PD1/LAG3 immune checkpoint blockade limits tumor development in a murine model of chronic lymphocytic leukemia. Blood 131, 1617-1621 (2018).

210. Nguyen, L. T. \& Ohashi, P. S. Clinical blockade of PD1 and LAG3-potential mechanisms of action. Nat. Rev. Immunol. 15, 45-56 (2015).

211. Huang, R. Y. et al. LAG3 and PD1 co-inhibitory molecules collaborate to limit CD8+ $T$ cell signaling and dampen antitumor immunity in a murine ovarian cancer model. Oncotarget 6, 27359-27377 (2015).

212. Woo, S. R. et al. Immune inhibitory molecules LAG-3 and PD-1 synergistically regulate T-cell function to promote tumoral immune escape. Cancer Res. 72, 917-927 (2012).

213. Hodi, F. S. et al. Improved survival with ipilimumab in patients with metastatic melanoma. N. Engl. J. Med. 363, 711-723 (2010).

214. Callahan, M. K. et al. Nivolumab plus ipilimumab in patients with advanced melanoma: updated survival, response, and safety data in a phase I doseescalation study. J. Clin. Oncol. 36, 391-398 (2018).

215. D'Angelo, S. P. et al. Nivolumab with or without ipilimumab treatment for metastatic sarcoma (Alliance A091401): two open-label, non-comparative, randomised, phase 2 trials. Lancet Oncol. 19, 416-426 (2018).

216. Hodi, F. S. et al. Nivolumab plus ipilimumab or nivolumab alone versus ipilimumab alone in advanced melanoma (CheckMate 067): 4-year outcomes of a multicentre, randomised, phase 3 trial. Lancet Oncol. 19, 1480-1492 (2018).

217. Kon, E. \& Benhar, I. Immune checkpoint inhibitor combinations: current efforts and important aspects for success. Drug Resist. Updat. 45, 13-29 (2019).

218. Tundo, G. R., Sbardella, D., Lacal, P. M., Graziani, G. \& Marini, S. On the horizon: targeting next-generation immune checkpoints for cancer treatment. Chemotherapy, 1-19, https://doi.org/10.1159/000500902 (2019)

219. Sznol, M. Long-term results encouraging for combination immunotherapy for advanced melanoma. ASCO 2014 (Chicago, 2014).

220. Sharma, P., Hu-Lieskovan, S., Wargo, J. A. \& Ribas, A. Primary, adaptive, and acquired resistance to cancer immunotherapy. Cell 168, 707-723 (2017).

221. Zappasodi, R., Merghoub, T. \& Wolchok, J. D. Emerging concepts for immune checkpoint blockade-based combination therapies. Cancer Cell 33, 581-598 (2018).

222. Duperret, E. K. et al. Synthetic DNA-encoded monoclonal antibody delivery of Anti-CTLA-4 antibodies induces tumor shrinkage in vivo. Cancer Res. 78, 6363-6370 (2018).

223. Ha, D. et al. Differential control of human Treg and effector T cells in tumor immunity by Fc-engineered anti-CTLA-4 antibody. Proc. Natl. Acad. Sci. USA 116, 609-618 (2019).

224. Sckisel, G. D. et al. Late administration of murine CTLA-4 blockade prolongs CD8-mediated anti-tumor effects following stimulatory cancer immunotherapy. Cancer Immunol. Immunother. 64, 1541-1552 (2015).

225. Verma, V. et al. PD-1 blockade in subprimed CD8 cells induces dysfunctional PD$1+$ CD38hi cells and anti-PD-1 resistance. Nat. Immunol. 20, 1231-1243 (2019).

226. Joller, N. \& Kuchroo, V. K. Tim-3, Lag-3, and TIGIT. Curr. Top. Microbiol. Immunol. 410, 127-156 (2017).

227. Johnson, D. B. et al. Ipilimumab therapy in patients with advanced melanoma and preexisting autoimmune disorders. JAMA Oncol. 2, 234-240 (2016).

228. Menzies, A. M. et al. Anti-PD-1 therapy in patients with advanced melanoma and preexisting autoimmune disorders or major toxicity with ipilimumab. Ann. Oncol. 28, 368-376 (2017).

229. Haanen, J. et al. Management of toxicities from immunotherapy: ESMO Clinical Practice Guidelines for diagnosis, treatment and follow-up. Ann. Oncol. 28, iv119-iv142 (2017).

230. Champiat, S. et al. Management of immune checkpoint blockade dysimmune toxicities: a collaborative position paper. Ann. Oncol. 27, 559-574 (2016).

231. Boutros, C. et al. Safety profiles of anti-CTLA-4 and anti-PD-1 antibodies alone and in combination. Nat. Rev. Clin. Oncol. 13, 473-486 (2016).

232. Postow, M. A., Sidlow, R. \& Hellmann, M. D. Immune-related adverse events associated with immune checkpoint blockade. N. Engl. J. Med. 378, 158-168 (2018).

233. Wang, D. Y. et al. Fatal toxic effects associated with immune checkpoint inhibitors: a systematic review and meta-analysis. JAMA Oncol. 4, 1721-1728 (2018).

234. Routy, B. et al. Gut microbiome influences efficacy of PD-1-based immunotherapy against epithelial tumors. Science 359, 91-97 (2018).

235. Elkrief, A., Derosa, L., Kroemer, G., Zitvogel, L. \& Routy, B. The negative impact of antibiotics on outcomes in cancer patients treated with immunotherapy: a new independent prognostic factor? Ann. Oncol. https://doi.org/10.1093/annonc/ mdz206 (2019).
236. Vetizou, M. et al. Anticancer immunotherapy by CTLA-4 blockade relies on the gut microbiota. Science 350, 1079-1084 (2015).

237. Torkamani, A., Wineinger, N. E. \& Topol, E. J. The personal and clinical utility of polygenic risk scores. Nat. Rev. Genet. 19, 581-590 (2018).

238. Gutierrez-Arcelus, M., Rich, S. S. \& Raychaudhuri, S. Autoimmune diseasesconnecting risk alleles with molecular traits of the immune system. Nat. Rev. Genet. 17, 160-174 (2016).

239. Lee, Y. et al. Induction and molecular signature of pathogenic TH17 cells. Nat. Immunol. 13, 991-999 (2012).

240. Ghoreschi, K. et al. Generation of pathogenic T(H)17 cells in the absence of TGFbeta signalling. Nature 467, 967-971 (2010).

241. McGeachy, M. J. et al. TGF-beta and IL- 6 drive the production of IL-17 and IL-10 by $\mathrm{T}$ cells and restrain $\mathrm{T}(\mathrm{H})-17$ cell-mediated pathology. Nat. Immunol. 8, 1390-1397 (2007).

242. Jager, A., Dardalhon, V., Sobel, R. A., Bettelli, E. \& Kuchroo, V. K. Th1, Th17, and Th9 effector cells induce experimental autoimmune encephalomyelitis with different pathological phenotypes. J. Immunol. 183, 7169-7177 (2009).

243. Nylander, A. N. et al. Podoplanin is a negative regulator of Th17 inflammation. JCl Insight 2, https://doi.org/10.1172/jci.insight.92321 (2017).

244. Peters, A. et al. Podoplanin negatively regulates CD4+ effector T cell responses. J. Clin. Invest. 125, 129-140 (2015).

245. Kishi, Y. et al. Protein $C$ receptor (PROCR) is a negative regulator of Th17 pathogenicity. J. Exp. Med. 213, 2489-2501 (2016).

246. Magnuson, A. M. et al. Identification and validation of a tumor-infiltrating Treg transcriptional signature conserved across species and tumor types. Proc. Natl. Acad. Sci. USA 115, E10672-E10681 (2018).

247. Schietinger, A. et al. Tumor-specific $T$ cell dysfunction is a dynamic antigendriven differentiation program initiated early during tumorigenesis. Immunity 45, 389-401 (2016)

248. Miller, B. C. et al. Subsets of exhausted CD8(+) T cells differentially mediate tumor control and respond to checkpoint blockade. Nat. Immunol. 20, 326-336 (2019).

249. Kurtulus, S. et al. Checkpoint blockade immunotherapy induces dynamic changes in PD-1(-)CD8(+) tumor-infiltrating T cells. Immunity 50, 181-194 e186 (2019).

250. Im, S. J. et al. Defining CD8 + T cells that provide the proliferative burst after PD1 therapy. Nature 537, 417-421 (2016).

251. $\mathrm{Wu}, \mathrm{T}$. et al. The TCF1-Bcl6 axis counteracts type I interferon to repress exhaustion and maintain T cell stemness. Sci. Immunol. 1, https://doi.org/ 10.1126/sciimmunol.aai8593 (2016).

252. Siddiqui, I. et al. Intratumoral Tcf1(+)PD-1(+)CD8(+) T cells with stem-like properties promote tumor control in response to vaccination and checkpoint blockade immunotherapy. Immunity 50, 195-211 e110 (2019).

253. Brummelman, J. et al. High-dimensional single cell analysis identifies stem-like cytotoxic CD8(+) T cells infiltrating human tumors. J. Exp. Med. 215, 2520-2535 (2018).

254. Miller, B. C. et al. Subsets of exhausted CD8+ T cells differentially mediate tumor control and respond to checkpoint blockade. Nat. Immunol. 20, 326-336 (2019).

255. Perez-Ruiz, E. et al. Prophylactic TNF blockade uncouples efficacy and toxicity in dual CTLA-4 and PD-1 immunotherapy. Nature 569, 428-432 (2019).

256. Willrich, M. A., Murray, D. L. \& Snyder, M. R. Tumor necrosis factor inhibitors: clinical utility in autoimmune diseases. Transl. Res. 165, 270-282 (2015).

257. Postow, M. A. \& Hellmann, M. D. Adverse events associated with immune checkpoint blockade. N. Engl. J. Med. 378, 1165 (2018).

258. Zhang, Y. et al. Hijacking antibody-induced CTLA-4 lysosomal degradation for safer and more effective cancer immunotherapy. Cell Res. 29, 609-627 (2019).

259. McGranahan, N. \& Swanton, C. Neoantigen quality, not quantity. Sci. Transl. Med. 11 (2019).

260. Nonomura, C. et al. Identification of a neoantigen epitope in a melanoma patient with good response to anti-PD-1 antibody therapy. Immunol. Lett. 208, 52-59 (2019).

261. Chen, F. et al. Neoantigen identification strategies enable personalized immunotherapy in refractory solid tumors. J. Clin. Invest. 129, 2056-2070 (2019).

262. June, C. H., O'Connor, R. S., Kawalekar, O. U., Ghassemi, S. \& Milone, M. C. CAR T cell immunotherapy for human cancer. Science 359, 1361-1365 (2018).

263. Chang, C. H. et al. Combination therapy with bispecific antibodies and PD-1 blockade enhances the antitumor potency of T Cells. Cancer Res. 77, 5384-5394 (2017).

264. Koopmans, I. et al. A novel bispecific antibody for EGFR-directed blockade of the PD-1/PD-L1 immune checkpoint. Oncoimmunology 7, e1466016 (2018).

265. Messaoudene, M. et al. T cell bispecific antibodies in node-positive breast cancer: novel therapeutic avenue for MHC class I loss variants. Ann. Oncol. https://doi.org/10.1093/annonc/mdz112 (2019).

266. Pai, C. S. et al. Tumor-conditional anti-CTLA4 uncouples antitumor efficacy from immunotherapy-related toxicity. J. Clin. Invest. 129, 349-363 (2019). 
267. Qu, H. Q. et al. Remapping the type I diabetes association of the CTLA4 locus. Genes Immun. 10(Suppl. 1), S27-S32 (2009).

268. Ban, Y. et al. Analysis of the CTLA-4, CD28, and inducible costimulator (ICOS) genes in autoimmune thyroid disease. Genes Immun. 4, 586-593 (2003).

269. Lei, C. et al. Association of the CTLA-4 gene with rheumatoid arthritis in Chinese Han population. Eur. J. Hum. Genet. 13, 823-828 (2005).

270. Brahmer, J. R. et al. Phase I study of single-agent anti-programmed death-1 (MDX-1106) in refractory solid tumors: safety, clinical activity, pharmacodynamics, and immunologic correlates. J. Clin. Oncol. 28, 3167-3175 (2010).

271. Lee, S. Y. \& Goverman, J. M. The influence of T cell Ig mucin-3 signaling on central nervous system autoimmune disease is determined by the effector function of the pathogenic T cells. J. Immunol. 190, 4991-4999 (2013).

272. Andrews, L. P., Marciscano, A. E., Drake, C. G. \& Vignali, D. A. LAG3 (CD223) as a cancer immunotherapy target. Immunol. Rev. 276, 80-96 (2017).
Open Access This article is licensed under a Creative Commons Attribution 4.0 International License, which permits use, sharing, adaptation, distribution and reproduction in any medium or format, as long as you give appropriate credit to the original author(s) and the source, provide a link to the Creative Commons license, and indicate if changes were made. The images or other third party material in this article are included in the article's Creative Commons license, unless indicated otherwise in a credit line to the material. If material is not included in the article's Creative Commons license and your intended use is not permitted by statutory regulation or exceeds the permitted use, you will need to obtain permission directly from the copyright holder. To view a copy of this license, visit http://creativecommons. org/licenses/by/4.0/.

(c) The Author(s) 2020 